Pirineos. Revista de Ecología de Montaña

vol. 176

Jaca, Enero-Diciembre, 2021, e067

ISSN-1: 0373-2568

https://doi.org/10.3989/pirineos.2021.176006

\title{
EFECTIVIDAD DE ÁREAS DE CONSERVACIÓN PRIVADA COMUNAL EN BOSQUES MONTANOS NUBLADOS DEL NORTE DEL PERÚ
}

\section{Effectiveness of Communal Privately Protected Areas in Monta- ne Cloud Forests of Northern Peru}

\footnotetext{
Ellen Delgado ${ }^{1,2 *}$, Gerson Meza Mori ${ }^{1}$, Elgar Barboza ${ }^{1,3}$, Nilton B. Rojas Briceño ${ }^{1}$, Cristóbal Torres Guzmán ${ }^{1}$, Manuel Oliva-Cruz', Segundo G. Chavez-Quintana ${ }^{1}$, Rolando Salas López ${ }^{1}$, Rocío López de la Lama ${ }^{4}$, C. Steven Sevillano-Ríos ${ }^{5}$, Fausto Sarmiento ${ }^{6}$

${ }^{1}$ Instituto de Investigación para el Desarrollo Sustentable de Ceja de Selva, Universidad Nacional Toribio Rodríguez de Mendoza de Amazonas, Chachapoyas 01001, Perú.

${ }^{2}$ Centro de Investigación Geoespacial, Departamento de Geografía, Universidad de Georgia, Athens, GA 30602 USA.

${ }^{3}$ Dirección de Desarrollo Tecnológico Agrario (DDTA), Instituto Nacional de Innovación Agraria (INIA), Av. La Molina 1981, Lima 15024, Perú.

${ }^{4}$ Institute for Resources, Environment and Sustainability at The University of British Columbia, 2202 Main Mall, Vancouver - BC, V6T 1Z4,

Canada.

${ }^{5}$ Centro de Ornitología y Biodiversidad - CORBIDI. C. 33, Santiago de Surco 15038, Lima, Perú

${ }^{6}$ Colaboratorio de Montología Neotropical, Departamento de Geografía, Universidad de Georgia, Athens, GA 30602 USA.
}

\author{
Identificador ORCID de los autores y e-mail \\ Ellen Delgado: https://orcid.org/0000-0002-4830-9280. E-mail: eldeflorian@uga.edu \\ Gerson Meza Mori: https://orcid.org/0000-0002-9754-6725. E-mail: gersonmmori@gmail.com \\ Elgar Barboza: https://orcid.org/0000-0002-9628-8138. E-mail: ebarboza@indes-ces.edu.pe \\ Nilton B. Rojas Briceño: https://orcid.org/0000-0002-5352-6140. E-mail: nrojas@indes-ces.edu.pe \\ Cristóbal Torres Guzmán: https://orcid.org/0000-0002-0932-7224. E-mail: cristobal.torres@untrm.edu.pe \\ Manuel Oliva-Cruz: https://orcid.org/0000-0002-9670-0970. E-mail: soliva@indes-ces.edu.pe \\ Segundo G. Chavez-Quintana: https://orcid.org/0000-0002-0946-3445. E-mail: segundo.quintana@untrm.edu.pe \\ Rolando Salas López: https://orcid.org/0000-0003-2184-6761. E-mail: rsalas@indes-ces.edu.pe \\ C. Rocio López de la Lama: https://orcid.org/0000-0001-6696-5401. E-mail: rocio.lopezdelalama@gmail.com \\ Steven Sevillano-Ríos: http://orcid.org/0000-0002-0642-5224. E-mail: ssevillano@corbidi.org \\ Fausto Sarmiento: http://orcid.org/0000-0003-0501-6020. Email: fsarmien@uga.edu \\ *Autor corresponsal: eldeflorian@uga.edu
}

Recibido: 11 de agosto de 2020. Aceptado: 26 de octubre de 2021. Fecha de publicación-on-line: 26 de noviembre de 2021 
Citation/cómo citar este artículo: Delgado, E., Meza Mori, G., Barboza, E., Rojas Briceño, N.B., Torres Guzmán, C., Oliva-Cruz, M., Chavez-Quintana, S.G., Salas López, R., López de la Lama, R., Sevillano-Ríos, C.S., Sarmiento, F. (2021). Efectividad de áreas de conservación privada comunal en bosques montanos nublados del norte de Perú. Pirineos, 176, e067. https://doi.org/10.3989/pirineos.2021.176006

RESUMEN: Las Áreas de Conservación Privada (ACP) son uno de los mecanismos de conservación, gestionadas por ciudadanos privados que más protagonismo han adquirido en los escenarios de conservación local en los últimos años. En este estudio evaluamos la efectividad de cuatro ACP gestionadas por comunidades locales (CC). Se aplicó el Índice de Efectividad Compuesto (IEC) para determinar la efectividad del diseño, la integridad ecológica y la gestión. Los resultados muestran sistemas de gestión con una efectividad media, tres de las cuatro ACP evaluados (Copallín, Huaylla Belén-Colcamar y Tilacancha) reportan un diseño efectivo. Los rangos altitudinales protegidos están entre 2500 y 3500 m.s.n.m., con un índice de representatividad de la superficie promedio de 4,55\% con respecto al área conservada en la categoría ACP para el departamento de Amazonas. La evaluación de la integridad ecológica indica que las ACP presentan menor superficie transformada (TS) (0-10\%) y mayor TS en sus áreas circundantes, especialmente en el ACP Tilacancha $(13,37 \%$ de TS en un buffer de 1,5 km). La suma ponderada de los índices individuales resulta en índices de efectividad compuestos de mayor a menor para el ACP Copallín $(2,22)$, Hierba Buena Allpayacku $(1,82)$, Huaylla Belen Colcamar $(1,81)$ y Tilacancha $(1,56)$.

PALABRAS CLAVE: Biodiversidad; ecosistemas de montaña; cambios de cobertura y uso del suelo (CCUS); análisis espacial; Yungas peruanas, Áreas Protegidas Privadas.

\begin{abstract}
Privately Protected Areas (PPA) are one of the conservation mechanisms managed by private citizens that have acquired more prominence in local conservation scenarios in recent years. In this study we evaluated the effectiveness of four (PPA) managed by local communities (CC). The Composite Effectiveness Index (CEI) was applied to determine the effectiveness of design, ecological integrity, and management. The results show management systems with medium effectiveness, three of the four PPA evaluated (Copallín, Huaylla Belén-Colcamar and Tilacancha) report effective design. The protected altitudinal ranges are between 2500 and 3500 m.a.s.l., with an average surface representativeness index of $4.55 \%$ with respect to the area conserved in the PPA category for the department of Amazonas. The evaluation of ecological integrity indicates that the PPA present lower transformed surface (TS) (0-10\%) and higher TS in their surrounding areas, especially in the Tilacancha PPA $(13.37 \%$ TS in a buffer of $1.5 \mathrm{~km})$. The weighted sum of the individual indices results in composite effectiveness indices from highest to lowest for the Copallín PPA (2.22), Hierba Buena Allpayacku (1.82), Huaylla Belen Colcamar (1.81) and Tilacancha (1.56).
\end{abstract}

KEY WORDS: Biodiversity; mountain ecosystems; changes in land cover and use (CCUS); spatial analysis; Peruvian Yungas, Privately Protected Areas.

\section{Introducción}

Las Áreas de Conservación Privada (ACP) son iniciativas de conservación gestionadas por ciudadanos privados y representan el $2 \%$ de las áreas protegidas globales (Bertzky et al., 2012). Son herramientas clave para la conservación a nivel local y regional. No obstante, permiten la participación de poblaciones locales y complementan los vacíos geográficos que puedan existir en las Áreas Naturales Protegidas de administración nacional (Stolton et al., 2014). Además, unifican los esfuerzos de conservación al incluir espacios que puedan ser refugios bioculturales de importancia local (Sarmiento et al., 2015).

En el Perú, las ACP son reconocidas en espacios con importancia biológica y ecosistémica, las cuales cuentan con un título de propiedad privada o comunal. Estas iniciativas de conservación al ser gestionadas por ciudadanos privados no reciben financiación por parte del Estado, pero complementan el Sistema Nacional de Áreas Naturales Protegidas por el Estado (SINANPE). En la actualidad se han creado más de 143 ACP que cubren 398.711 ha (Castillo et al., 2020; SERNANP, 2020). El 46\% de ACP son gestionadas por personas individuales o familias, el
$36 \%$ por comunidades campesinas (CC) y el $18 \%$ por empresas privadas y Organizaciones no Gubernamentales (ONG). Las ACP han cobrado importancia en los últimos años en escenarios de conservación local, pero aún continúan estando subrepresentadas en informes internacionales de mecanismos de conservación (Stolton et al., 2014), por lo cual, existe una necesidad de evidenciar las características de estos espacios de conservación en diferentes contextos socioambientales y aplicar metodologías que permitan dar información sobre su efectividad.

Los estudios disponibles referentes a evaluación de la efectividad de áreas protegidas incluyen metodologías diversas basadas en el análisis de la cobertura de hábitat y poblaciones de especies (Geldmann et al., 2013) y la evaluación de la deforestación y los cambios de cubierta y uso del suelo (CCUS) dentro y en la zona circundante del área de conservación en un determinado espacio y tiempo (Figueroa et al., 2011; Figueroa \& Sánchez-Cordero, 2008; Andam et al., 2008; Spracklen et al., 2015). Otras metodologías aplicaron un índice consolidado de los componentes de diseño, planificación, integridad ecológica y gestión (Andreasen et al., 2001; Lee \& Abdullah, 2019). Si bien las evaluaciones de las áreas de conservación disponibles pro- 
porcionan información valiosa a escalas globales, los resultados se ven comprometidos en los análisis sub-globales, debido a las particularidades ecológicas y regulatorias de cada territorio. Por tanto, junto con las pruebas de campo, se necesitan análisis espacial a escala local, para determinar si las áreas de conservación como las ACP, cumplen sus objetivos de creación (Castillo et al., 2020).

En este estudio se aplicó el índice de efectividad compuesto en cuatro ACP administradas por CC del Norte del Perú. Para ello, se determinó subíndices de (i) efectividad de diseño, basado en la representatividad ecosistémica y de elevación, (ii) integridad ecológica, mediante la evaluación de los CCUS, y (iii) efectividad de gestión, a través de la aplicación de encuestas a los representantes de los comités de gestión de cada ACP. Los tres subíndices se integraron mediante suma lineal ponderada utilizando pesos a partir de un Proceso Analítico Jerárquico (AHP). Los resultados brindan un marco metodológico que permite la evaluación rápida de la efectividad de las ACP.

\section{Materiales y Métodos}

\section{1. Área de estudio}

El departamento de Amazonas $\left(3^{\circ} 0^{\prime}-7^{\circ} 2^{\prime}\right.$ Sur, $77^{\circ} 0^{\prime}-$ $78^{\circ} 42^{\prime}$ Oeste, $120-4.900$ m.s.n.m y 39,25 mil km²), ubicado en el Norte de los Andes peruanos (Figura 1), alberga una variedad de especies de flora y fauna únicas del bosque montano nublado (MINAM, 2014a). Es el departamento con mayor superficie protegida (145.458,78 ha) mediante ACP en Perú (SERNANP, 2020). Cuenta con 19 ACP, 13 de ellas gestionadas por CC y seis por asociaciones o particulares. Por un lado, las ACP pequeñas son propiedad de empresarios urbanos que autofinancian la conservación a través del ecoturismo. Por otro lado, las ACP extensas son gestionadas por comunidades rurales, cuyos medios de vida dependen de la agricultura y la ganadería. Mientras que los gerentes de las ACP pequeñas tienden a permanecer durante muchos años, los gerentes de ACP extensas dependen de consejos comunales elegidos democráticamente que cambian periódicamente, lo que conduce a una falta de continuidad en la gestión (Mitchell et al., 2018). Por ello, una gran cantidad de ACP comunales aún no han zonificado el uso del suelo (limitado o múltiple) o delimitado sus fronteras. Incluso los propios comuneros no conocen los límites del ACP, lo que aumenta la vulnerabilidad de estos espacios ante actividades antrópicas (Monteferri, 2019).

En este estudio se seleccionaron cuatro ACP del departamento de Amazonas, en base a (i) mayor antigüedad de creación (año 2011), (ii) titularidad a nombre de CC y (iii) que representen más del $50 \%$ del tipo de cobertura de una ecorregión. Todo esto con la finalidad de comparar las características ambientales y agroproductivas de las mismas. Las ACP seleccionadas fueron ACP Copallín, ACP Hierba Buena-Allpayacku, ACP Huaylla Belén-Colcamar y ACP Tilacancha (Figura 1a-d). En suma, representan el
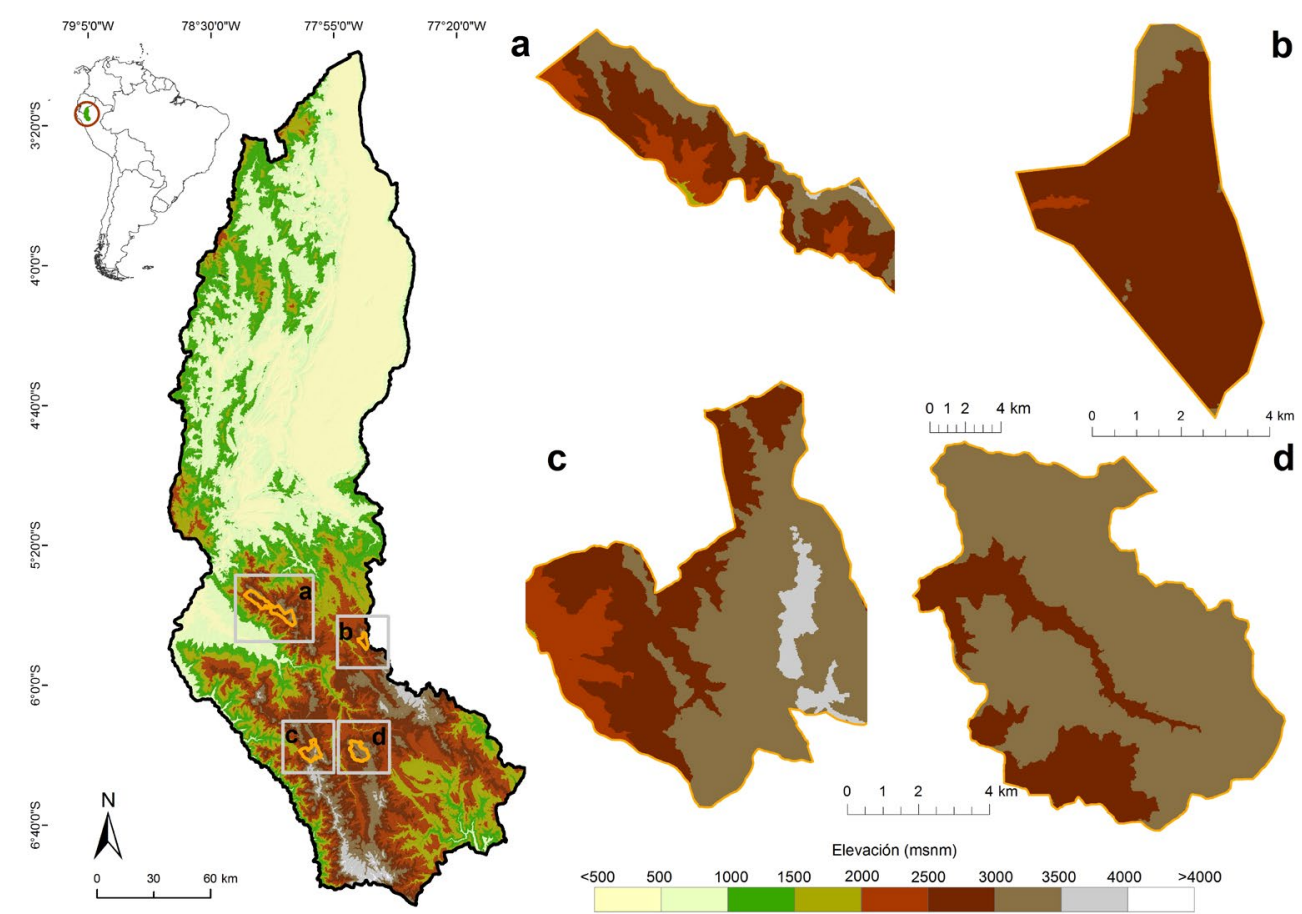

Figura 1. Ubicación de las ACP en el departamento de Amazonas: a) Copallín, b) Hierba Buena- Allpayacku c) Huaylla Belén Colcamar d) Tilacancha.

Figure 1. Location of the ACP in the Department of Amazonas: a) Copallin, b) Hierba Buena-Allpayacku c) Huaylla Belén Colcamar d) Tilacancha. 
$18,2 \%$ del territorio protegido por ACP en Amazonas y ubicándose todas ellas en la ecorregión Yungas Peruanas (León et al., 2006) (Tabla 1). En todos los casos, más del $70 \%$ del área de estudio se encuentra dentro del territorio de CC. Esto permitió involucrar a actores locales en la evaluación de la efectividad de las ACP bajo un enfoque participativo y de gestión del territorio.

\subsection{Diseño metodológico}

Se diseñó un Índice de Efectividad Compuesto (IEC) para cada ACP a partir del Índice de Efectividad de Diseño (IED), del Índice de Efectividad Ecológica (IEE) y del Índice de Efectividad de gestión (IEG) (Figura 2).

\section{3. Índice de Efectividad de la Gestión (IEG)}

Este índice permitió evaluar si la administración del ACP se ejecuta en base a una planificación definida, verificando si cumple con el propósito de su creación, o encontrando puntos débiles en la gestión. Para el cálculo del IEG, se adaptó lo propuesto por Ramírez (2015) en la Metodología para la Evaluación y Priorización Rápidas del Manejo de Áreas Protegidas (RAPPAM) (Ervin, 2003). Esta metodología se aplicó mediante una encuesta dividida en cinco criterios, y cada criterio estuvo compuesto por varios indicadores. Los criterios e indicadores (entre paréntesis) fueron: Contexto (importancia biológica, importancia socio-económica, vulnerabilidad, políticas de las áreas protegidas, ambiente político), Planificación (objetivos, seguridad legal, diseño del sitio y

Tabla 1. Características de las ACP evaluadas en el departamento de Amazonas

Table 1. Characteristics of the evaluated ACP in the Department of Amazonas

\begin{tabular}{|c|c|c|c|c|c|c|}
\hline ACP & $\begin{array}{c}\text { Resolución } \\
\text { ministerial de } \\
\text { reconocimiento }\end{array}$ & Vigencia & $\begin{array}{c}\text { Comunidad } \\
\text { Campesina } \\
\text { titular }\end{array}$ & Área (ha) & $\begin{array}{c}\text { Superficie } \\
\text { conservada } \\
\text { respecto a } \\
\text { Amazonas (\%) }\end{array}$ & $\begin{array}{c}\text { Tipo de } \\
\text { ecorregión }\end{array}$ \\
\hline Copallín & $\begin{array}{c}\text { RM } \\
140-2011-M I N A M\end{array}$ & Perpetuidad & Copallín & $11.549,21$ & 7,79 & $\begin{array}{c}\text { Yungas } \\
\text { peruanas }\end{array}$ \\
\hline $\begin{array}{c}\text { Hierba Buena- } \\
\text { Allpayacku }\end{array}$ & $\begin{array}{c}\text { RM } \\
123-2011-M I N A M\end{array}$ & Perpetuidad & Corosha & $2.282,12$ & 1,54 & $\begin{array}{c}\text { Yungas } \\
\text { peruanas }\end{array}$ \\
\hline $\begin{array}{c}\text { Huaylla Belén- } \\
\text { Colcamar }\end{array}$ & $\begin{array}{c}\text { RM } \\
166-2011-M I N A M\end{array}$ & 30 años & Colcamar & $6.338,42$ & 4,28 & $\begin{array}{c}\text { Yungas } \\
\text { peruanas }\end{array}$ \\
\hline Tilacancha & $\begin{array}{c}\text { RM } \\
118-2010-M I N A M\end{array}$ & 20 años & Levanto y Mayno & $6.800,48$ & 4,59 & $\begin{array}{c}\text { Yungas } \\
\text { peruanas }\end{array}$ \\
\hline
\end{tabular}

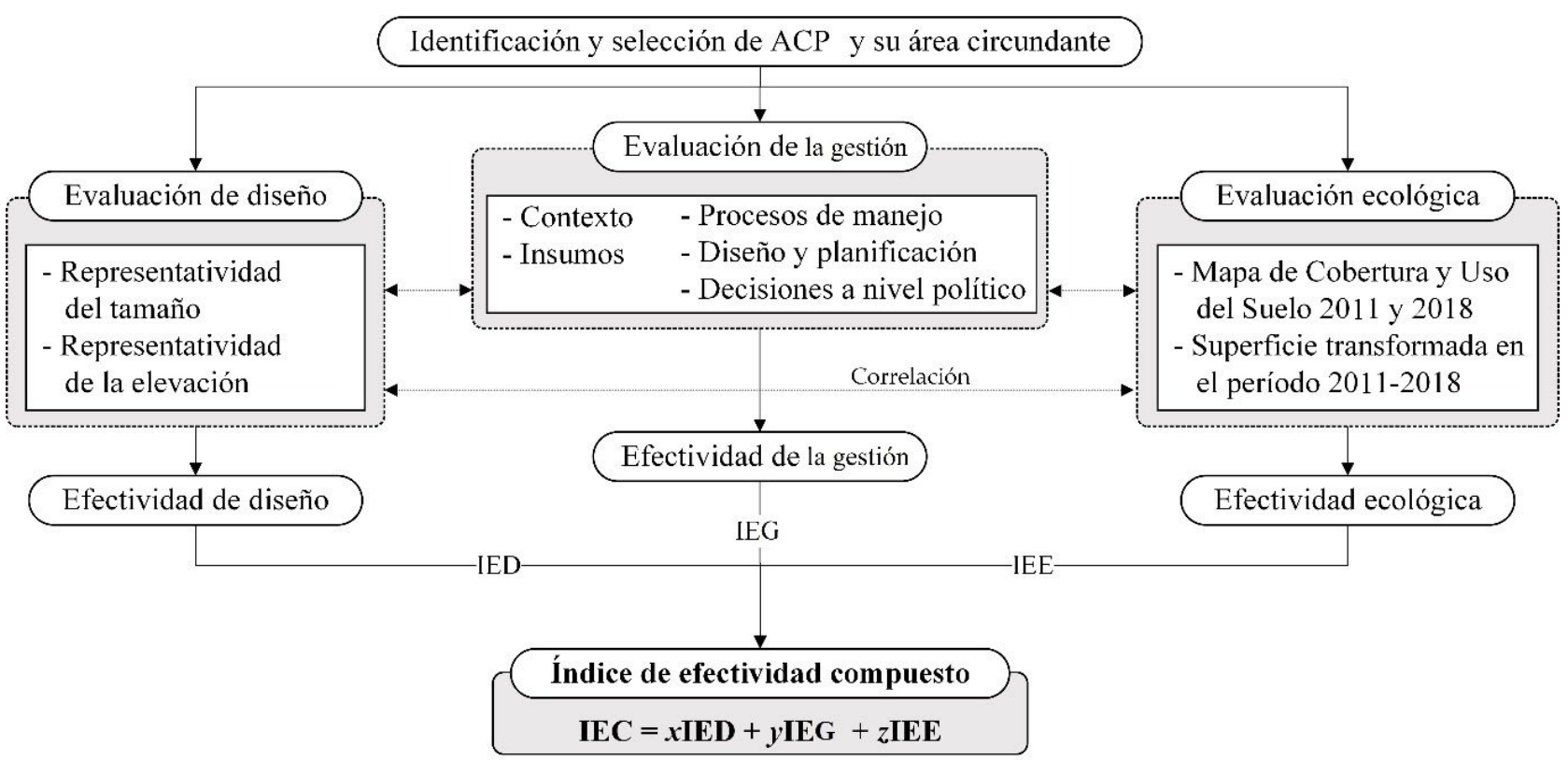

Figura 2. Flujograma metodológico para generar el Índice de Efectividad Compuesto para ACP en el departamento de Amazonas, basado en Lee \& Abdullah (2019).

Figure 2. Methodological flow chart to generate the Composite Effectiveness Index for ACP in the department of Amazonas, based on Lee \& Abdullah (2019). 
planificación, diseño del subsistema de áreas protegidas), Insumo (asignación de personal, comunicación e información, infraestructura y equipo, finanzas), Procesos (planificación de la gestión, toma de decisiones para la gestión, investigación, monitoreo y evaluación) y Resultados (restauración de zonas degradadas o deforestadas, manejo de fauna silvestre, extensión comunitaria, construcción de infraestructura, investigación, monitoreo, manejo de visitantes y aplicación de estrategias de adaptación al cambio climático).

La encuesta consistió en 132 preguntas y cada una se calificó con una escala tipo Likert, es decir, de 1 a 4 puntos, donde 1 es bajo, 2 es moderado, 3 es medio y 4 es alto (Rodríguez-Rodríguez \& Martínez-Vega, 2012). La calificación de cada indicador se obtuvo mediante promedio de las valoraciones de las preguntas dentro de cada indicador que, a su vez, permitió obtener el IEG (escala de 1 a 4). Las personas encuestadas fueron conocedoras de la gestión del ACP tal como lo sugiere Lee \& Abdullah (2019). Así, por ejemplo, se entrevistó a integrantes de los comités de gestión vigentes y anteriores, así como pobladores de las comunidades locales asentadas en el área de influencia directa de las ACP. Además, se realizaron visitas a las poblaciones asentadas en las zonas circundantes de las ACP para complementar la interpretación de los datos de la encuesta. La selección de los participantes comunales se realizó mediante un muestreo no probabilístico "bola de nieve" (Naderifar et al., 2017).

\section{4. Índice de Efectividad de Diseño (IED)}

El IED evalúa de qué manera la representatividad, el tamaño y la localización del ACP cubren el rango máximo de biodiversidad protegida (Lee \& Abdullah, 2019). Para determinar el IED se consideraron dos subíndices (Hazen \& Anthamatten, 2004): el de representatividad de superficie (CIs), y el de representatividad de elevación (CIe).

Se calculó el CIs dividiendo la superficie del ACP respecto a la superficie total de ACP del departamento de Amazonas. La información espacial de cada ACP se descargó del geoservidor del SERNANP (http://geo.sernanp. gob.pe/visorsernanp/). Para calcular el CIe se utilizó el Modelo de Elevación Digital (DEM SRTM, 30 m) descargado del portal del Servicio Geológico de los Estados Unidos (https://earthexplorer.usgs.gov/). El DEM, fue ecotado según los límites del departamento de Amazonas. A continuación, se clasificó en nueve clases con rangos cada $500 \mathrm{~m}$, calculando el porcentaje de todas las ACP por cada clase de elevación. Luego, se obtuvo el cociente entre el porcentaje del ACP en cada clase de elevación sobre el porcentaje de todas las ACP del departamento. Para cada ACP se calculó el promedio del CIe de los cocientes anteriores. Finalmente, el IED se promedió entre CIs y CIe. Un valor IED $>1$ denotó una buena representación, mientras que un IED $<1$ implicó una representación pobre en términos de rangos, tanto altitudinales como espaciales (Trisurat, 2007).

\section{5. Índice de Efectividad Ecológica (IEE)}

La evaluación de la integridad ecológica implica un análisis cuantitativo de la capacidad que tienen las áreas de conservación para mantener a largo plazo las condiciones necesarias que permiten la existencia de la biodiversidad que protegen (Ervin, 2003). Para el IEE se sugiere la evaluación de la conectividad, fragmentación, hemerobia e idoneidad del hábitat (Lee \& Abdullah, 2019). No obstante, nuestro objetivo fue trabajar con un índice de aplicación rápida y de bajo coste. En consecuencia, estudiamos los procesos de CCUS, utilizados comúnmente para evaluar la integridad ecológica debido a la repercusión en la dinámica del ecosistema y a su relación con otros procesos de deterioro ambiental (Sánchez-Cordero et al., 2005). Si bien no todo proceso de CCUS implica una reducción de la integridad ecológica, los procesos de gran magnitud pueden inducir la modificación de la estructura y el funcionamiento de los ecosistemas (Turner et al., 2007).

El IEE se evaluó siguiendo la metodología descrita por Figueroa \& Sánchez-Cordero (2008) y aplicada por Figueroa et al. (2011), la cual genera un índice en base al análisis de los CCU del ACP y su área circundante, como una aproximación (cf., proxy) de la existencia de alta biodiversidad en sistemas naturales y de poca diversidad en las zonas influenciadas por el cambio. Para cada ACP, el área circundante se delimitó con un tamaño proporcional al ACP. Se generó un área de amortiguamiento (cf., buffer) de 6.399,60 ha para el ACP Huaylla Belén-Colcamar, 6.495,25 ha para el ACP Tilacancha, 12.165,84 ha para el ACP Copallín y 2.607,89 ha para el ACP Hierba Buena-Allpayacku.

Para elaborar los mapas de CCUS se siguió el flujograma metodológico propuesto por Rojas et al., 2019. Se utilizaron imágenes del satélite Landsat 5 y 8, con resolución espacial de 30 metros de 2011 y 2018. Estas fueron adquiridas de la plataforma del Servicio Geológico de los Estados Unidos (USGS) (https://earthexplorer.usgs.gov/) y corregidas atmosféricamente utilizando el Semi-Automatic Classification Plugin (SCP) (Congedo, 2013). Para las ACP Copallín y ACP Hierba Buena Allpayacku, altamente afectadas por nubosidad, se realizaron composiciones libres de nubes utilizando imágenes de hasta dos años en Google Earth Engine (Gorelick et al., 2017).

Las imágenes multiespectrales se clasificaron, utilizando el algoritmo de Máxima Verosimilitud en ArcMap 10.7 en base a firmas espectrales de áreas de entrenamiento recolectadas en recorridos en campo en cada ACP. La exactitud temática de los mapas se evaluó con una Matriz de Confusión basada en 196 sitios de verificación (Chuvieco, 2016). Estos sitios fueron establecidos a través de un muestreo aleatorio sistemático, no alineado, estratificado sobre el mapa final clasificado (MINAM, 2014b). Luego fueron verificados en campo, en Google Earth Pro (v. 7.3.0.3832) y SAS Planet (v. 160707) (Rojas et al., 2019), y se calculó la Exactitud Global y el Índice de Kappa (k) (Story \& Congalton, 1986).

Se calculó la tasa de cambio de la superficie transformada (ST) para cada ACP, entre 2011 y 2018, mediante la 
(Ecuación 1) (Figueroa \& Sánchez-Cordero, 2008), donde $\mathrm{S}_{1}$ y $\mathrm{S}_{2}$ son las superficies de cobertura y uso del suelo (CUS) en la fecha $\mathrm{t} 1 \mathrm{y} \mathrm{t} 2$, respectivamente.

$$
\mathrm{ST}=1-\left(\mathrm{S}_{2} / \mathrm{S}_{1}\right)^{1 /(\mathrm{t} 2-\mathrm{t} 1)}
$$

(Ecuación 1)

Se obtuvo un IEE para cada ACP a partir de los valores descritos en la (Ecuación 2). Los últimos dos parámetros (D, DE) únicamente adquieren valores de 0 o 1 : cuando las ACP presentan un incremento en la ST mayor que su respectiva área circundante o ecorregión adquieren un valor de 0 ; en caso contrario, adquieren un valor de 1 . El IEE, por lo tanto, puede tomar valores entre 0 y 4 . Las ACP con valores en el índice cercanos a 0 son de baja efectividad, al tener valores reducidos en varios parámetros, en tanto que las ACP con valores cercanos a 4 serían las más efectivas del conjunto de AP analizado (Figueroa et al., 2011).

$$
\mathrm{IEE}=\mathrm{S}+\mathrm{ST}+\mathrm{D}+\mathrm{DE}
$$

(Ecuación 2)

S: Porcentaje ocupado por superficies transformadas dentro del ACP en 2018.

D: Diferencia entre la ST en el ACP y la ST en sus áreas circundantes.

DE: Diferencia entre la ST en el ACP y la ST en sus respectivas ecorregiones.

\section{6. Índice de Efectividad Compuesto (IEC)}

Para generar el IEC, con base en una suma lineal ponderada de los IED, IEE e IEM, se requiere asignar un peso de importancia a cada índice (x, y, z, en la Figura 2) (Lee
\& Abdullah, 2019). Para determinar esos pesos se aplicaron dos enfoques: (i) el mismo peso para los tres índices (1/3) o promedio y (ii) diferentes pesos, calculados mediante Matrices de Comparación por Pares (PCM). Las PCM permiten comparar un índice respecto a los demás y establecer un grado de importancia entre sí (Mighty, 2015). La comparación se basó en la escala de nueve niveles de (Saaty, 1977) (Tabla 2), donde cada miembro de un grupo de expertos asignó un juicio de valor, de menos a más importante, según su experiencia y la realidad local. Las PCM cuadradas fueron desarrolladas por expertos profesionales con experiencia en la gestión para la creación y gestión de Áreas de Conservación en el Perú, comunidades locales que lideran iniciativas de conservación en el Perú, y académicos con intereses en la investigación para la conservación de recursos naturales en los Andes Tropicales. Las PCM se complementaron con columnas que comprenden las etapas de normalización, priorización (estandarización de criterios) y cálculos de verificación de consistencia de la matriz (Yunis et al., 2020). La priorización permite obtener el Peso de Importancia de cada índice, donde la suma debe ser igual a 1. En el desarrollo de las PCM cuadradas, las preferencias subjetivas de los expertos pueden generar inconsistencias en la priorización. Por tanto, para definir el valor aceptable para esta inconsistencia, se calculó la Relación de Consistencia (CR) (Saaty, 1990). La CR es el cociente entre el Índice de Consistencia (CI) de la matriz en cuestión y un Índice de Consistencia Aleatorio (RI). El RI está definido según el número de columnas de la matriz (n) y con tres índices (n $=3$ ), el RI es 0,525 (Aguarón \& Moreno-Jiménez, 2003). El CI depende del valor propio más grande o principal de la matriz $(\lambda \max )$ y n (Ecuación 3$)$.

$$
C I=(\lambda \max -n) /(n-1)
$$

(Ecuación 3)

Tabla 2. Escala establecida para la asignación de los juicios de valor en las PCM. Table 2. Scale established for the assignment of value judgments in the PCM

\begin{tabular}{|c|c|c|c|c|c|c|c|c|}
\hline $1 / 9$ & $1 / 7$ & $1 / 5$ & $1 / 3$ & 1 & 3 & 5 & 7 & 9 \\
\hline Extrema & Fuerte & \multicolumn{2}{|c|}{ Moderada } & \multirow{2}{*}{ Igual } & \multicolumn{2}{|c|}{ Moderada } & Fuerte & Extrema \\
& \multicolumn{3}{|c|}{ Más importante } \\
\hline
\end{tabular}

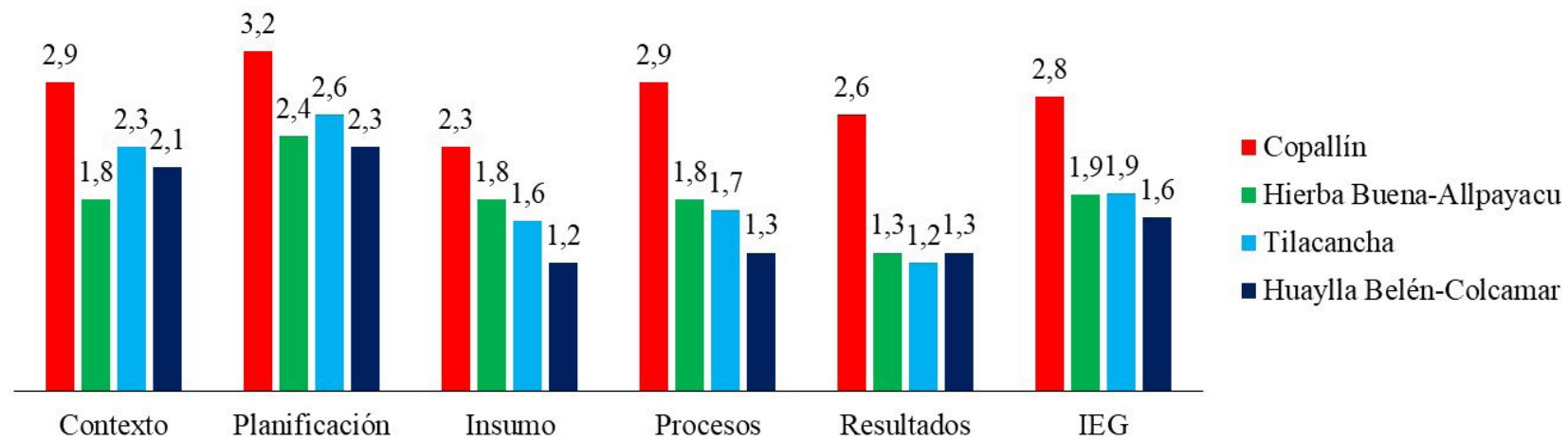

Figura 3. Puntuaciones por cada criterio evaluado en la efectividad de la gestión de las ACP.

Figure 3. Scores for each criterion evaluated in the effectiveness of ACP management. 


\section{Resultados}

\subsection{Efectividad de la gestión}

El ACP Copallín fue la más efectiva en cuanto a gestión en todos los criterios evaluados en comparación con las demás ACP. Los resultados del índice de gestión de mayor a menor fueron: Copallín $(2,8)$, Tilacancha $(1,9)$, Hierba Buena- Allpayacku $(1,9)$, y Huaylla Belén $(1,6)$ (Tabla S1; Figura 3). En base a los rangos establecidos (de 1 a 4), en general, todas las ACP evaluadas reportan una efectividad de gestión que oscila entre baja a moderada, a excepción de Copallín con una efectividad de gestión de moderada a media.

El común denominador de las ACP con mayor efectividad en la gestión son los valores más altos alcanzados en cuanto a planificación, contexto y procesos. El contexto valoró la importancia biológica, la vulnerabilidad del ecosistema, así como la importancia socioeconómica por parte de las comunidades locales. Los valores máximos y mínimos fueron obtenidos por Tilacancha y Hierba Buena-Allpayacku, con 2,89 y 1,84 , respectivamente. Estos resultados difieren entre sí debido a la valoración realizada por parte de las comunidades locales a las políticas del $\mathrm{ACP}$, así como el ambiente político que rige la conservación a nivel local.

Las CC de Copallín, Tilacancha y Hierba Buena-Allpayacku estaban más involucradas en la gestión del ACP y obtuvieron mayores puntajes en el componente de planificación. En contraste, Huaylla Belén-Colcamar obtuvo la puntuación más baja, donde incluso no se encontró un sistema de gestión implementado a nivel de comunidad ni del comité de gestión. Los comités de gestión de las ACP como Copallín, Tilacancha y Hierba Buena-Allpayacku reportaron haber accedido a mayores fondos económicos y asesoramiento técnico por parte de instituciones externas (principalmente ONGs). Estos recursos fueron empleados en la realización de actividades sostenibles en el área protegida, mejorar procesos de gestión, y ejecutar acciones de respuesta rápida ante amenazas. Hecho que se ve reflejado en mayores puntuaciones en el componente insumos para las tres áreas mencionadas. En el componente resultados se evaluó la disminución de amenazas en el área y la persistencia de objetos de conservación. Aquí, el ACP Copallín obtuvo el mayor puntaje de evaluación $(2,6)$.

\subsection{Efectividad de diseño}

La Figura 4 y Tabla S2 presentan el IED y sus subíndices para cada ACP evaluada en Amazonas. Las ACP Copallín y Tilacancha mostraron los valores máximos de CIs, con 2,2 y 1,3, respectivamente y el ACP Hierba Buena- Allpayacku mostró el valor más bajo $(0,4)$. Con respecto a la CIe, el ACP Tilacancha reporta mayor representatividad de elevación con respecto a las ACP evaluadas. En general, todas las ACP mostraron un IED adecuado, a excepción de Hierba Buena-Allpayacku que reportó un IED bajo (0.8).

\subsection{Integridad ecológica}

Se elaboraron ocho mapas de CUS (Figura S1) que estuvieron representados por las clases de bosque, pajonal y pastos y cultivos, los cuales presentaron una exactitud global de 0,78 a 0,88, y un índice de Kappa de 0,69 a 0,77, respectivamente. El bosque y pajonal representó la mayor proporción en las ACP de Copallín, Hierba Buena- Allpayacku y Huaylla Belén-Colcamar. En la ACP Tilacancha predominaron el pajonal y pastos y cultivos. De la superposición cartográfica entre los mapas de 2011 y 2018 de cada ACP, la Figura 5 muestra procesos de los CCUS. Las ACP Tilacancha, Hierba Buena- Allpayacku y Huaylla Belén-Colcamar presentan el mayor cambio con respecto a la ACP Copallín.

Por otro lado, se observan diferentes estados de conservación de cubierta natural (pajonal andino y bosque). En todas las ACP el porcentaje de la superficie transformada es generalmente bajo (0-10\%). Sin embargo, aun cuando todas las ACP muestran un buen estado de con-

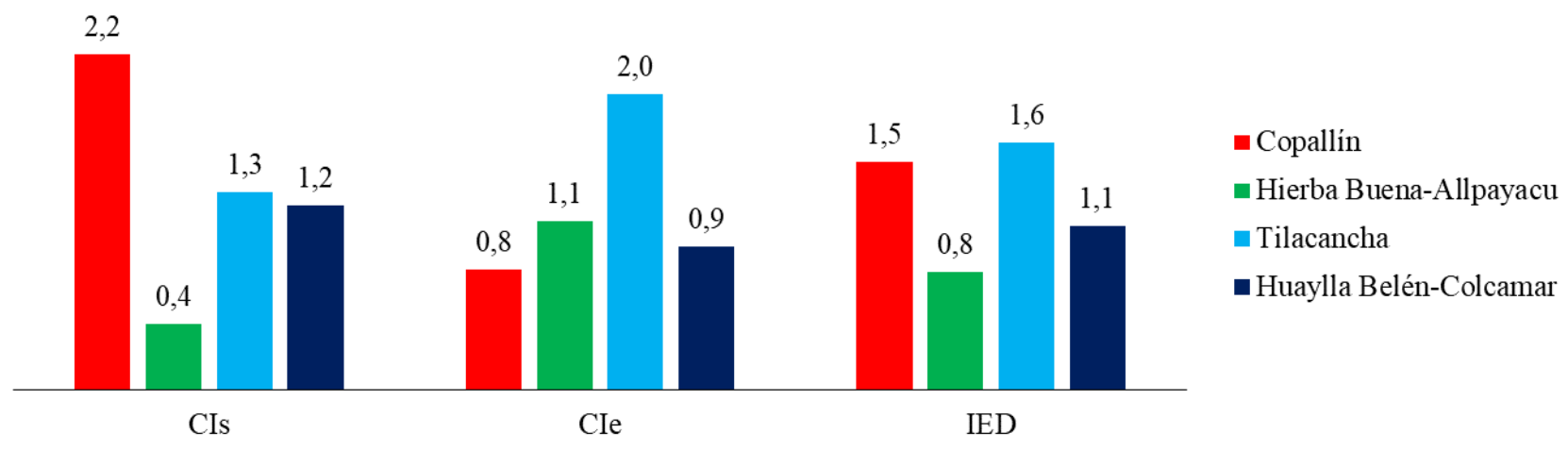

Figura 4. Puntuaciones de los subindices CIs, CIe e IED.

Figure 4. Scores in the subindex Cis, Cle and IED. 

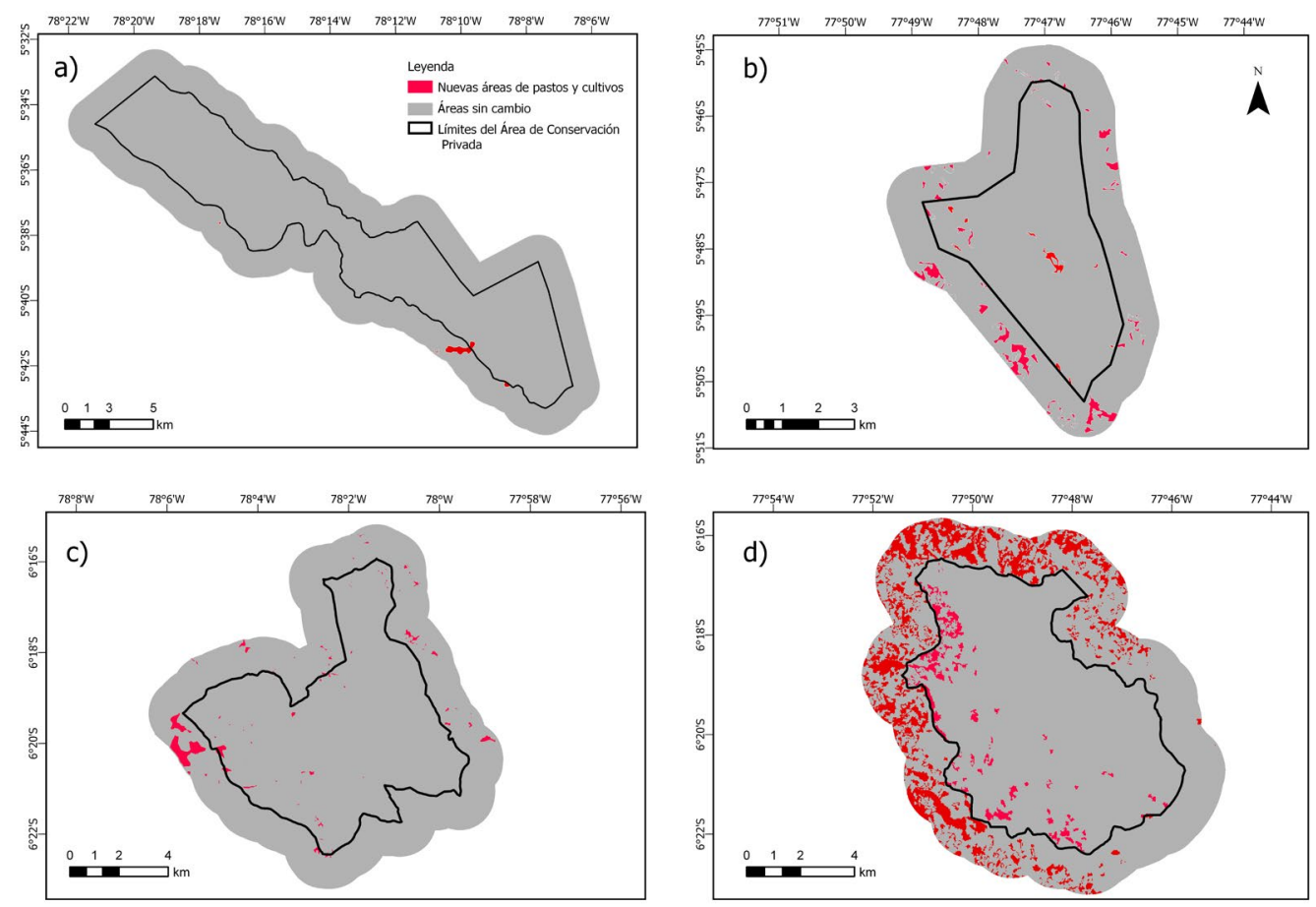

Figura 5. Procesos de CCUS entre 2011-2018 en las ACP (a) Copallín, (b) Hierba Buena- Allpayacku, (c) Huaylla Belén-Colcamar y (d) Tilacancha, en Amazonas (Perú).

Figure 5. CCUS processes between 2011-2018 in the ACP(a) Copallin, (b) Hierba Buena-Allpayacku, (c) Huaylla Belén-Colcamar y (d) Tilacancha, in Amazonas (Perú).

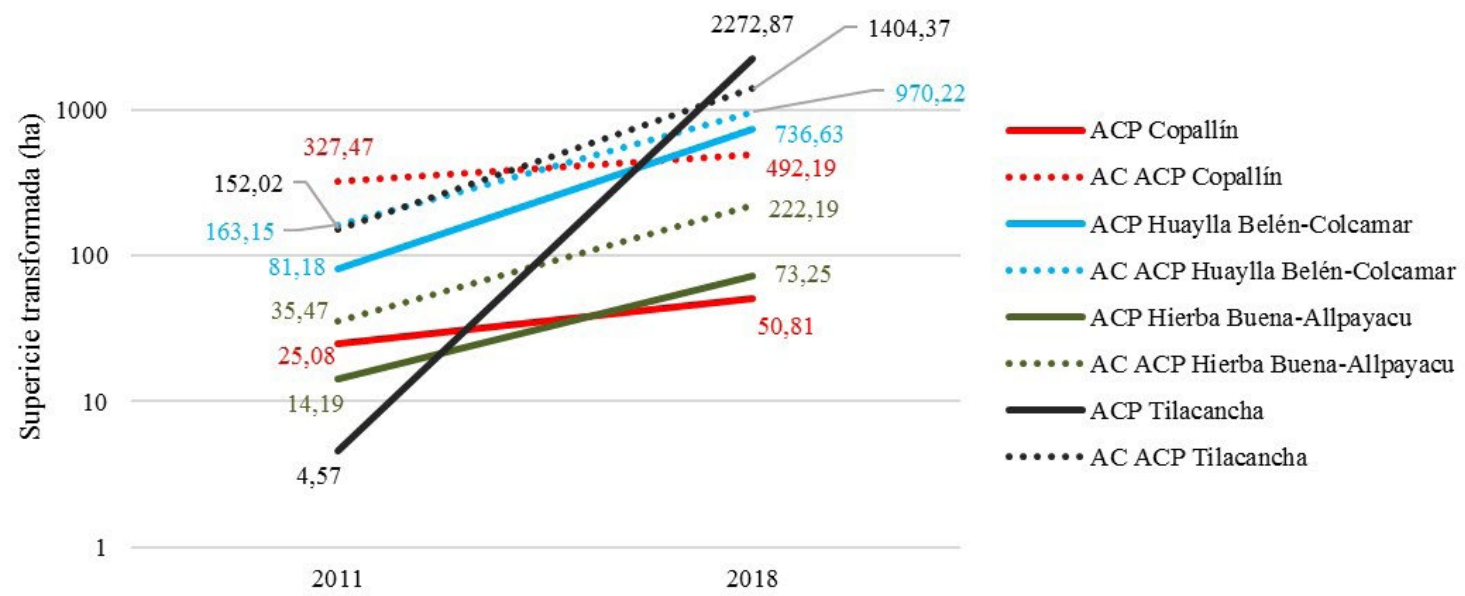

Figura 6. Superficie transformada (ha/año) en las ACP y su área circundante en 2011 y 2018. Figure 6. Transformed area (ha/year) in the ACPs and the surrounding area in 2011 and 2018.

servación, las áreas analizadas han experimentado un aumento de su superficie transformada con una tasa media de cambio neto de 10 ha/año (Figura 6). Con relación a la extensión de cada ACP, los valores máximos de superficie transformada en el 2018 se observaron en el ACP Tilacancha y Huaylla Belén-Colcamar con 163 ha y 152 ha, respectivamente, lo cual representa el 2,17 y el 1,29\% de la superficie total de las áreas. En contraste, en el ACP
Hierba Buena Allpayacku, se observaron menos cambios con una ST neta de 3 ha/año, lo cual representa el 0,93\% del territorio del ACP.

En general, las cuatro ACP mostraron menores ST que sus respectivas áreas circundantes (AC). Sin embargo, algunas ACP reportaron mayores niveles ST en sus áreas circundantes que otras, lo cual influyó en el valor del IEE que obtuvieron. Cabe resaltar que estas diferencias son relativas 
Tabla 3. Peso de importancia de índices de efectividad y ratios de consistencia de las PCM.

Table 3. Weight of importance of effectiveness indices and consistency ratios of the PCM

\begin{tabular}{|c|c|c|c|c|c|c|c|c|c|}
\hline \multirow{2}{*}{$\begin{array}{l}\text { Índice / } \\
\text { Ratio }\end{array}$} & \multicolumn{7}{|c|}{ Expertos } & \multirow{2}{*}{$\begin{array}{c}\text { Peso } \\
\text { promedio }\end{array}$} & \multirow{2}{*}{$\begin{array}{c}\text { Peso } \\
\text { normalizado }\end{array}$} \\
\hline & 1 & 2 & 3 & 4 & 5 & 6 & 7 & & \\
\hline IED & 0,20 & 0,09 & 0,30 & 0,51 & 0,16 & 0,06 & 0,53 & 0,14 & 0,1556 \\
\hline IEE & 0,71 & 0,17 & 0,90 & 0,36 & 0,25 & 0,65 & 0,10 & 0,43 & 0,4777 \\
\hline IEM & 0,09 & 0,74 & 0,08 & 0,13 & 0,59 & 0,29 & 0,37 & 0,33 & 0,3667 \\
\hline$\lambda \max$ & 3,10 & 3,01 & 3,05 & 3,10 & 3,05 & 3,08 & 3,09 & & \\
\hline CI & 0,05 & 0,01 & 0,03 & 0,05 & 0,03 & 0,04 & 0,05 & & \\
\hline CR & 0,09 & 0,01 & 0,50 & 0,09 & 0,05 & 0,08 & 0,09 & & \\
\hline
\end{tabular}

ya que dependen del tamaño y de los procesos de CCUS que ocurren en cada ACP. En este sentido, las ACP Huaylla Belén-Colcamar, Hierba Buena-Allpayacku y Copallín mostraron menores niveles de ST en sus áreas circundantes, y obtuvieron un IEE de 2,64, 2,60 y 2,71 respectivamente, en una escala del 1-4. Esto indica un alto grado de efectividad para prevenir los procesos de CCUS. Mientras que el ACP Tilacancha, que obtuvo el mayor nivel ST en su AC (13\%) obtuvo el valor más bajo de IEE $(1,98)$.

\section{4. Índice de Efectividad Compuesto (IEC)}

Se construyeron siete PCM, una por cada uno de los siete expertos en ACP que colaboraron con el estudio. La Tabla 3 indica los pesos de importancia de los índices de efectividad y ratios de consistencia de la PCM de cada experto. Todas las matrices resultaron consistentes $(\mathrm{CR}<0,10)$ y se usaron para obtener el peso de importancia promedio de cada índice.
El IEC1 para cada ACP se calculó utilizando el mismo peso para los tres índices (o promedio de IEM, IED e IEE), mientras que el IEC2 se calculó utilizando la función lineal (Ecuación 4) con los pesos respectivos encontrados a partir de las PCM procesadas en la (Tabla S3). Para IEC1, el límite inferior calculado fue de 1,59 a 2,10, calculados a partir de modelos simulados con los valores mínimos y máximos que puede obtenerse en las ACP del departamento de Amazonas. De manera análoga, los límites inferior y superior para IEC2 fueron 1,56 y 2,22 para las ACP Tilacancha y Copallín, respectivamente. Empleando la Ecuación 4, se calcularon los IEC para cada ACP. El índice de efectividad está compuesto con los pesos obtenidos por PCM (IEC1) y el IEC por adición simple (IEC2), conforme se presenta en la Figura 7 y Tabla S4.

$$
\begin{gathered}
\mathrm{IEC}=(0,1556) \mathrm{IED}+(0,4777) \mathrm{IEE}+(0,3667) \mathrm{IEG} \\
(\text { Ecuación } 4)
\end{gathered}
$$

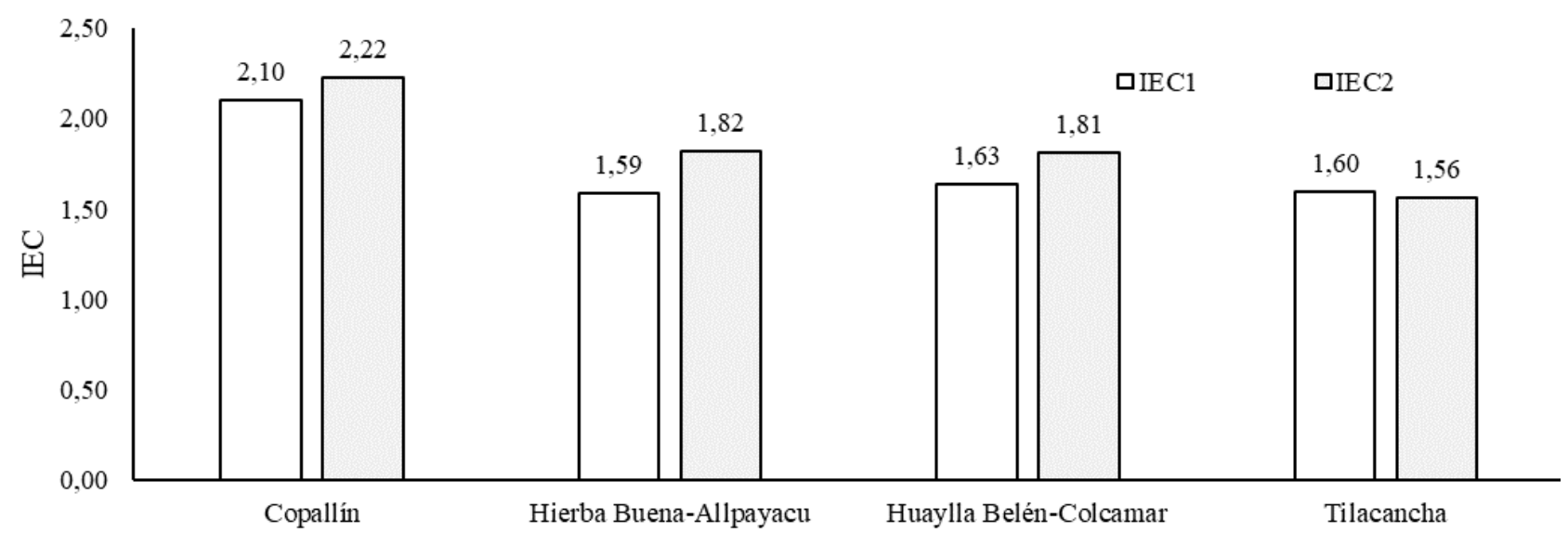

Figura 7. Índice de efectividad compuesto de las ACP evaluadas en Amazonas (Perú). Figure 7. Effectiveness Index composed of the evaluated ACP in Amazonas (Perú). 


\section{Discusión}

Esta investigación permitió conocer la efectividad de las ACP estudiadas y generar información clave a partir de la construcción de un IEC, el cual incluye aspectos ecológicos, de diseño y gestión. Este enfoque permitió encontrar relaciones entre los componentes evaluados e identificar las causas de la efectividad o debilidades en las ACP. Más aún, esta metodología constituye una ventaja sobre las evaluaciones estándar de efectividad de áreas de conservación, que se centran en aspectos de gestión (Coad et al., 2015; Dudley et al., 2004; Hockings et al., 2008; Kurdoğlu \& Çokçalişkan, 2011; López-Rodríguez \& Rosado, 2017).

La mayor efectividad de gestión estuvo representada por las ACP Copallín y Hierba Buena - Allpayacku. Las razones derivan de que estas ACP presentaron una institucionalidad sólida en la comunidad, comités de gestión activos y presencia de actores locales involucrados en actividades de conservación. Esto va de la mano con la literatura que enfatiza la importancia del capital social, la gestión impulsada desde las bases y el desarrollo de normas y sanciones por parte de la propia comunidad para la sostenibilidad de las iniciativas de conservación (Agrawal, 2005).

Por otro lado, las ACP con los niveles más bajos de efectividad de gestión fueron Tilacancha y Colcamar, que presentaron comités de gestión poco organizados y capacitados, principalmente afectados por el cambio de representantes cada año. Estas ACP presentan sistemas de administración y gobernanza compartida entre comunidades con jurisdicciones diferentes. Esto podría indicar que los límites del sistema socio-ecológico no están bien definidos, lo que implica competencia por los recursos naturales e incumplimiento de normas comunes establecidas (Berkes et al., 1989; Ostrom et al., 1999).

En general, las cuatro ACP evaluadas reportaron niveles de medio a bajo en los indicadores de insumos y resultados. Así, la ACP Copallín presentó un nivel medio. Por su parte, las ACP Hierba Buena-Allpayacku, Tilacancha y Huaylla Belén-Colcamar presentaron niveles bajos en ambos indicadores. Estos niveles dependen directamente de la financiación económica de las ACP, que fueron asignadas en la etapa de creación y los primeros años de gestión, sin mayor alcance, lo cual afecta a la efectividad de gestión. Esto podría sugerir que existe una relación directa entre la cantidad de insumos que manejan las ACP con los resultados obtenidos.

La evaluación de la efectividad de diseño muestra que las ACP Copallín, Tilacancha y Huaylla Belén-Colcamar cuentan con buena representación respecto a la distribución de la superficie protegida, mientras que la ACP Hierba Buena-Allpayacku tiene baja efectividad de diseño. Esto se debe a que IED evalúa la proporción de elevación y superficie conservada con respecto a un punto de referencia regional o nacional (Hazen \& Anthamatten, 2004). Estos resultados indican que el objetivo principal de las ACP en ecosistemas de Yungas Peruanas en Amazonas es proteger la flora y fauna en cabeceras de cuencas hidrográficas, por lo cual, en su mayoría se encuentran localizadas sobre los 2500 m.s.n.m con una superficie promedio de 2500 ha. Este hecho corroboraría que las ACP responden a las necesidades de conservación a escala local, ubicados en ecosistemas transformados (Baldwin \& Fouch, 2018; Bargelt et al., 2020).

Asimismo, las ACP han sido eficientes para mantener su integridad ecológica, como reporta la reducción de los CCUS dentro de sus límites. Sin embargo, las amenazas externas son constantes, lo que impacta en pérdidas de hábitats en las áreas circundantes de las ACP. Una de las razones por la cual las áreas circundantes se encuentran más afectadas, es la historicidad del paisaje y el tipo de aprovechamiento permitido dentro y fuera de los límites del ACP como la agricultura migratoria y la ganadería extensiva, comunes en ecosistemas de ceja de selva (Mendoza et al., 2017; Rojas et al., 2019; Salas et al., 2016; Sarmiento, 2020). Lo mismo ocurre con el impacto directo en los hábitats de la vida silvestre en diferentes áreas protegidas (Jiang \& Yu, 2019). Esto condiciona la disponibilidad de fuentes de agua e incrementa la presión en el área protegida y las especies endémicas de bosques nubosos (Shanee et al., 2013).

Los IEC calculados para las cuatro ACP, permitieron elaborar un ranking de efectividad. Tal como sugieren Lee \& Abdullah (2019), la normalización de las puntuaciones y la inclusión de las ponderaciones facilita la interpretación del IEC puesto que ajustan los valores a una escala entre 0 y 1 . La normalización de los índices obtenidos para cada ACP debe realizarse teniendo en cuenta los límites máximos y mínimos posibles para cada componente del IEC. Para determinar el rango de IED se calculó los valores para todas las ACP del departamento de Amazonas, por lo que se sugiere simplificar su cálculo de acuerdo con la zona de estudio.

La principal ventaja de presentar los resultados en un IEC es brindar la solidez del valor del índice y proporcionar una referencia precisa a los responsables de la formulación de políticas, los tomadores de decisiones u otras partes interesadas en la conservación de los recursos naturales. Además, el análisis de los índices únicos y la verificación de las correlaciones entre los diferentes factores que influyen en la efectividad pueden ayudar a identificar las causas del problema en la gestión, diseño e integridad ecológica y proporcionar una solución más clara para el ACP (Lee \& Abdullah, 2019). Por ello, es importante analizar individualmente los resultados obtenidos por cada subíndice individual y componente evaluado. Finalmente, la aplicación de esta metodología en contextos similares debe realizarse teniendo en cuenta las características ambientales de la zona de estudio y el contexto histórico y cultural que determina los usos y conservación de los recursos naturales (Sarmiento \& Sarmiento 2021).

\section{Conclusiones}

El IEC que evalúa aspectos de la gestión, diseño y la integridad ecológica de las cuatro ACP seleccionadas, muestra sistemas de gestión con una efectividad media. 
Las principales limitaciones de gestión están relacionadas con factores de (1) planificación del presupuesto, (2) equipamiento, (3) personal para monitoreo y (4) vigilancia y control de amenazas. Entre las amenazas identificadas destacan la deforestación por agricultura itinerante, los incendios forestales, la tala selectiva y la pérdida de fauna representativa. Tres de las cuatro áreas evaluadas (Copallín, Huaylla Belén-Colcamar y Tilacancha) poseen un diseño efectivo. Los rangos altitudinales protegidos más representativos para las cuatro ACP en estudio se encuentran entre los 2500 y 3500 m.s.n.m. La evaluación de la integridad ecológica indica menores niveles de superficie transformada dentro de los límites de las ACP y mayores en las áreas circundantes, producto del desarrollo de actividades agropecuarias.

La metodología propuesta permitió realizar el análisis de la efectividad de las ACP en términos de gestión, diseño e integridad ecológica. Además, del estudio de los factores que influyen en el alcance de la gestión de las comunidades locales en las ACP y su dinámica en áreas circundantes. Por otro lado, las técnicas de cartografía participativa como uso de mapas temáticos, imágenes satelitales y visitas al área de estudio con miembros de las comunidades locales, contribuyeron a la exploración de los ecosistemas en bosques de neblina del norte del Perú.

\section{Otras consideraciones}

Estudios futuros podrían orientar la evaluación de componentes del ecosistema como la conectividad, calidad del hábitat y funcionalidades para mantener los objetivos de conservación. La aplicación de métricas, como la correlación de Pearson, puede examinar la relación entre cada componente o indicador y el IEC evaluado. Asimismo, se recomienda ampliar el estudio al conjunto de ACP que conforman la red de conservación voluntaria del Perú, a fin de encontrar tendencias y relaciones de la diversidad geográfica, participación social y modelos de gestión en la mejora de la efectividad de este tipo de iniciativas de conservación. Finalmente, es indispensable la continuidad de estos estudios para mejorar los procesos de gestión de las ACP por parte de las comunidades locales; en este sentido, la participación de instituciones académicas, estatales y no gubernamentales es de suma importancia.

\section{Agradecimientos}

Los autores agradecen al Instituto de Investigación para el Desarrollo Sustentable de Ceja de Selva (INDES-CES) de la Universidad Nacional Toribio Rodríguez de Mendoza de Amazonas (UNTRM), que a través del proyecto BIODIVERSIDAD (SNIP No. 316114) hizo posible la financiación de la investigación. Asimismo, agradecemos al Colaboratorio de Montología Neotropical de la Universidad de Georgia (USA) y al proyecto VULPES (NSF ICER-1624207), por su participación en esta publicación y por hacer posible la pasantía de estudios donde se hizo el análisis de datos y la revisión de estudios relacionados a la gestión de áreas protegidas a nivel internacional. Al Banco Mundial que financió al Programa Nacional de Innovación Agraria (PNIA) del Instituto Nacional de Innovación Agraria (INIA), que contribuyó con el desarrollo de esta investigación. Finalmente, agradecemos al trabajo minucioso de dos revisores anónimos y a Fernando Corroto por la revisión final, quienes mejoraron la calidad de este manuscrito.

\section{Referencias}

Agrawal, A., 2005. Community, Intimate Government, and the Making of Environmental Subjects in Kumaon, India. Environmentality, 46(2): 161-190. http://www.jstor.org/stable/10.1086/427122

Aguarón, J. \& Moreno-Jiménez, J.M., 2003. The geometric consistency index: Approximated thresholds. European Journal of Operational Research, 147: 137-145. https://doi. org/10.1016/S0377-2217(02)00255-2

Andam, K.S., Ferraro, P.J., Pfaff, A., Sanchez-Azofeifa, G.A. \& Robalino, J.A., 2008. Measuring the effectiveness of protected area networks in reducing deforestation. Proceedings of the National Academy of Sciences of the United States of America, 105(42): 16089-16094. https://doi.org/10.1073/ pnas. 0800437105

Andreasen, J.K., O’Neill, R.V., Noos, R. \& Slosser, N.C., 2001. Considerations for the development of a terrestrial index of ecological integrity. Ecological Indicators, 1: 21-35. https://doi.org/10.1016/S1470-160X(01)00007-3

Baldwin, R.F. \& Fouch, N.T., 2018. Understanding the Biodiversity Contributions of Small Protected Areas Presents Many Challenges. Land, 7(123): 1-12. https://doi.org/10.3390/ land 7040123

Bargelt, L., Fortin, M.J. \& Murray, D.L., 2020. Assessing connectivity and the contribution of private lands to protected area networks in the United States. PLoS ONE, 15(3): 1-13. https://doi.org/10.1371/journal.pone.0228946

Berkes, F., Feeny, D., McCay, B.J. \& Acheson, J.M., 1989. The benefits of the commons. Nature, 340(6229): 91-93.

Bertzky, B., Corrigan, C., Kemsey, J., Kenney, S., Ravilious, C., Besançon, C.y Burgess, N. (2012). Protected Planet Report 2012: Tracking progress towards global targets for protected areas. Gland, Switzerland and Cambridge, UK: IUCN and UNEP-WCMC. https://2012congress.iucn.org/cmsdata.iucn. org.iucn.vm.iway.ch/downloads/protected planet_report.pdf

Castillo, L.S., Correa Ayram, C.A., Matallana Tobón, C.L., Corzo, G., Areiza, A., González, M.R., Serrano, F., Chalán Briceño, L., Sánchez Puertas, F., More, A., Franco, O., Bloomfield, H., Aguilera Orrury, V.L., Rivadeneira Canedo, C., Morón-Zambrano, V., Yerena, E., Papadakis, J., Cárdenas, J.J., Golden Kroner, R.E. \& Godínez-Gómez, O., 2020. Connectivity of Protected Areas: Effect of Human Pressure and Subnational Contributions in the Ecoregions of Tropical Andean Countries. Land 2020, 9: 239. https://doi. org/10.3390/land9080239

Chuvieco, E., 2016. Fundamentals of satellite remote sensing. An environmental approach (CRC Press Taylor \& Francis Group; 2da ed.).

Coad, L., Leverington, F., Knights, K., Geldmann, J., Eassom, A., Kapos, V., Kingston, N., Lima, M. De, Zamora, C., Cuardros, I., Nolte, C., Burgess, N.D., Hockings, M. \& Kapos, V., 2015. Measuring impact of protected area management interventions: current and future use of the Global Database of Protected Area Management Effectiveness. Philosophical 
Transactions of the Royal Society B: Biological Sciences, 370 (1681): 20140281.

Congedo, L. (2013). Semi-Automatic Classification Plugin for QGIS. Rome: Sapienza University. http://www.academia. edu/download/34540581/WP-SemiAutomatic_Classification_Plugin_for_QGIS.pdf

Dudley, N., Bēokurov, A., Borodin, O., Higgins-Zogib, L., Hockings, M., Lacerda, L. \& Stolton, S., 2004. How Effective are Protected Areas? WWF International.

Ervin, J., 2003. Rapid assessment of protected area management effectiveness in four countries. BioScience, 53(9): 833-841. https://doi.org/10.1641/0006-3568(2003)053[0833:RAOPAM]2.0.CO;2

Figueroa, F. \& Sánchez-Cordero, V., 2008. Effectiveness of natural protected areas to prevent land use and land cover change in Mexico. Biodiversity and Conservation, 17(13): 3223-3240. https://doi.org/10.1007/s10531-008-9423-3

Figueroa, F., Sánchez-Cordero, V., Illoldi-Rangel, P. \& Linaje, M., 2011. Evaluation of protected area effectiveness for preventing land use and land cover changes in Mexico. Is an index good enough? Revista Mexicana de Biodiversidad, 82(3): 951-963. https://doi.org/10.22201/ ib.20078706e.2011.3.768

Geldmann, J., Barnes, M., Coad, L., Craigie, I.D., Hockings, M. \& Burgess, N.D., 2013. Effectiveness of terrestrial protected areas in reducing habitat loss and population declines. Biological Conservation, 161: 230-238. https://doi.org/10.1016/j. biocon.2013.02.018

Gorelick, N., Hancher, M., Dixon, M., Ilyushchenko, S., Thau, D. \& Moore, R., 2017. Google Earth Engine: Planetary-scale geospatial analysis for everyone. Remote Sensing of Environment, 202(2016): 18-27. https://doi.org/10.1016/j. rse.2017.06.031

Hazen, H.D. \& Anthamatten, P.J., 2004. Representation of Ecological Regions by Protected Areas at the Global Scale. Physical Geography, 25(6): 499-512. https://doi. org/10.2747/0272-3646.25.6.499

Hockings, M., James, R., Stolton, S., Dudley, N., Mathur, V., Makombo, J., Courrau, J. \& Parrish, J., 2008. Enhancing our Heritage Toolkit Assessing management effectiveness of natural World Heritage sites (UNESCO World Heritage Centre (ed.)).

Jiang, L. \& Yu, L, 2019. Analyzing land use intensity changes within and outside protected areas using ESA CCI-LC datasets. 20(July 2018). https://doi.org/10.1016/j.gecco.2019.e00789

Kurdoğlu, O. \& Cokçalişkan, B.A., 2011. Assessing the effectiveness of protected area management in the Turkish Caucasus. African Journal of Biotechnology, 10(75): 1720817222. https://doi.org/10.5897/AJB11.2204

Lee, W.H. \& Abdullah, S.A., 2019. Framework to develop a consolidated index model to evaluate the conservation effectiveness of protected areas. Ecological Indicators, 102: 131-144. https://doi.org/10.1016/j.ecolind.2019.02.034

León, B., Pitman, N. \& Roque, J., 2006. Introducción a las plantas endémicas del Perú. 13(2).

López-Rodríguez, F. \& Rosado, D., 2017. Management effectiveness evaluation in protected areas of southern Ecuador. Journal of Environmental Management, 190: 45-52. https:// doi.org/10.1016/j.jenvman.2016.12.043

Mendoza, M.E., Salas, R. \& Barboza, E., 2017. Análisis multitemporal de la deforestación usando la clasificación basada en objetos, distrito de Leymebamba (Perú). INDES Revista de Investigación Para El Desarrollo Sustentable, 3(2): 67-76. https://doi.org/10.25127/INDES.20153.210

Mighty, M.A., 2015. Site suitability and the analytic hierarchy process: How GIS analysis can improve the competitive advantage of the Jamaican coffee industry. Applied Geography, 58: 84-93. https://doi.org/https://doi.org/10.1016/j. apgeog.2015.01.010
MINAM, 2014a. Perú reino de bosques (Geobosques (ed.); 1st ed.). http://www.bosques.gob.pe/archivo/1455ad_perureinodebosques.pdf

MINAM, 2014b. Protocolo: Evaluacion de la Exactitud Tematica del Mapa de Deforestación. In: Ministerio del Ambiente. $\mathrm{http} / /$ www.minam.gob.pe/ordenamientoterritorial/wp-content/uploads/sites/18/2013/10/Protocolo-Validacion-Mapa-Deforestacion.pdf

Mitchell, B.A., Stolton, S., Bezaury-Creel, J., Bingham, H.C., Cumming, T.L., Dudley, N., Fitzsimons, J.A., Malleret-King, D., Redford, K.H. \& Solano, P., 2018. Guidelines for privately protected areas. Best practice protected area guidelines series (Issue 29). https://www.iucn.org/es/content/areas-bajo-proteccion-privada-mirando-al-futuro

Monteferri, B., 2019. Áreas de conservación privada en el Perú: Avances y propuestas a 20 años de su creación.

Naderifar, M., Goli, H. \& Ghaljaie, F., 2017. Snowball Sampling: A Purposeful Method of Sampling in Qualitative Research. Strides Development of Medical Education, 14(3): 1-6. https://doi.org/10.5812/sdme.67670.

Ostrom, E., Burger, J., Field, C.B., Norgaard, R.B. \& Policansky. D., 1999. Revisiting the Commons: Local Lessons, Global Challenges. Science, 284(5412): 278-282. https://doi. org/10.1126/science.284.5412.278

Ramírez, M., 2015. Evaluación de la efectividad de manejo en las áreas silvestres protegidas del área de conservación Tempisque. Central Agronómico Tropical de Investigación y Enseñanza.

Rodríguez-Rodríguez, D. \& Martínez-Vega, J., 2012. Proposal of a system for the integrated and comparative assessment of protected areas. Ecological Indicators, 23(2012): 566-572. https://doi.org/10.1016/j.ecolind.2012.05.009

Rojas, N.B., Barboza, E., Maicelo, J.L., Oliva, S.M. \& Salas, R., 2019. Deforestation in the peruvian Amazon: Indexes of Land Cover/Land Use (LC/LU) changes based on GIS. Boletin de la Asociacion de Geografos Espanoles, 81: 1-34. https://doi.org/10.21138/bage.2538a

Saaty, T.L., 1977. A scaling method for priorities in hierarchical structures. Journal of Mathematical Psychology, 15(3): 234-281. https://doi.org/10.1016/0022-2496(77)90033-5

Saaty, T.L., 1990. How to make a decision: The Analytic Hierarchy Process. European Journal of Operational Research, 48: 9-26. https://doi.org/10.1016/0377-2217(90)90057-I

Salas, R., Barboza, E. \& Oliva, S.M., 2016. Dinámica multitemporal de índices de deforestación en el distrito de Florida, Departamento de Amazonas, Perú. INDES Revista de Investigación Para El Desarrollo Sustentable, 2(1): 18-27. http:// revistas.untrm.edu.pe/index.php/INDES/article/view/59/175

Sánchez-Cordero, V., Illoldi-Rangel, P., Linaje, M., Sarkar, S. \& Townsend, A., 2005. Deforestation and extant distributions of Mexican endemic mammals. Biological Conservation, 126: 465-473. https://doi.org/10.1016/j.biocon.2005.06.022

Sarmiento, F.O., 2020. Montology manifesto: echoes towards a transdisciplinary science of mountains. Journal of Mountain Science, 17(10): 2512-2527. https://doi.org/https://doi. org/10.1007/s11629-019-5536-2

Sarmiento, F.O \& Sarmiento, E.V., 2021. Flancos Andinos: Paleoecología, Biogeografía Crítica y Ecología Política en los Climas Cambiantes de los Bosques Neotropicales de Monta$\tilde{n} a$. Editorial INDES CES/UNTRM. Universidad Nacional Toribio Rodríguez de Mendoza, Amazonas. Chachapoyas, Peru. https://www.doi.org/10.5281/zenodo.4746912

Sarmiento, F.O., Bernbaum, E., Lennon, J. \& Brown, J., 2015. Managing cultural uses and features. In: Worboys, G. et al. (editors). IUCN's Protected Area Governance and Management. ANU Press. Sydney, Australia. Pp. 686-714. https:// doi.org/10.22459/PAGM.04.2015.22 
SERNANP, 2020. Información geográfica de las Áreas Naturales Protegidas del Perú. http://geo.sernanp.gob.pe/visorsernanp/

Shanee, N., Hotspot, B. \& Martin, S., 2013. Campesino justification for self-initiated conservation actions : a challenge to mainstream conservation. Journal of Political Ecology, 20: 413-428. https://doi.org/10.2458/v20i1.21754

Spracklen, B.D., Kalamandeen, M., Galbraith, D., Gloor, E. \& Spracklen, D.V., 2015. A global analysis of deforestation in moist tropical forest protected areas. PLoS ONE, 10(12): 1-16. https://doi.org/10.1371/journal.pone.0143886

Stolton, S., Redford, K.H., William, D., Adams, B.M. \& Corcuera, E., 2014. Areas Bajo Protección Privada: Mirando al Futuro (UINC (ed.); Issue 1). https://www.iucn.org/es/content/areas-bajo-proteccion-privada-mirando-al-futuro
Story, M. \& Congalton, R.G., 1986. Accuracy Assessment : A User's Perspective. Photogrammetric Engineering and Remote Sensing, 52(3): 397-399.

Trisurat, Y., 2007. Applying Gap Analysis and a Comparison Index to Evaluate Protected Areas in Thailand: 235-245. https://doi.org/10.1007/s00267-005-0355-3

Turner, B.L., Lambin, E.F. \& Reenberg, A., 2007. The emergence of land change science for global environmental change and sustainability. Proceedings of the National Academy of Sciences, 104(52): 20666-20671. https://doi.org/10.1073/ pnas.0704119104

Yunis, C.R.C., López, R.S., Cruz, S.M.O., Castillo, E.B., López, J.O.S., Trigoso, D.I. \& Briceño, N. B.R., 2020. Land suitability for sustainable aquaculture of rainbow trout (Oncorhynchus mykiss) in molinopampa (Peru) based on RS, GIS, and AHP. ISPRS International Journal of Geo-Information, 9(1). https://doi.org/10.3390/ijgi9010028 
Pirineos. Revista de Ecología de Montaña

vol. 176

Jaca, Enero-Diciembre, 2021, e067

ISSN-1: 0373-2568

\title{
Efectividad de Áreas de Conservación Privada Comunal en Bosques Montanos Nublados del Norte del Perú
}

\section{Effectiveness of Communal Privately Protected Areas in Montane Cloud Forests of Northern Peru}

\footnotetext{
Ellen Delgado ${ }^{1,2 *}$, Gerson Meza Mori ${ }^{1}$, Elgar Barboza ${ }^{1,3}$, Nilton B. Rojas Briceño ${ }^{1}$, Cristóbal Torres Guzmán ${ }^{1}$, Manuel Oliva-Cruz. ${ }^{1}$, Segundo G. Chavez-Quintana ${ }^{1}$, Rolando Salas López ${ }^{1}$, Rocío López de la Lama ${ }^{4}$, C. Steven Sevillano-Ríos ${ }^{5}$, Fausto Sarmiento ${ }^{6}$

${ }^{1}$ Instituto de Investigación para el Desarrollo Sustentable de Ceja de Selva, Universidad Nacional Toribio Rodríguez de Mendoza de Amazonas, Chachapoyas 01001, Perú.

${ }^{2}$ Centro de Investigación Geoespacial, Departamento de Geografía, Universidad de Georgia, Athens, GA 30602 USA.

${ }^{3}$ Dirección de Desarrollo Tecnológico Agrario (DDTA), Instituto Nacional de Innovación Agraria (INIA), Av. La Molina 1981, Lima 15024, Perú.

${ }^{4}$ Institute for Resources, Environment and Sustainability at The University of British Columbia, 2202 Main Mall, Vancouver - BC, V6T 1Z4, Canada.

${ }^{5}$ Centro de Ornitología y Biodiversidad - CORBIDI. C. 33, Santiago de Surco 15038, Lima, Perú

${ }^{6}$ Colaboratorio de Montología Neotropical, Departamento de Geografía, Universidad de Georgia, Athens, GA 30602 USA.
}

\author{
Identificador ORCID de los autores y e-mail \\ Ellen Delgado: https://orcid.org/0000-0002-4830-9280. E-mail: eldeflorian@uga.edu \\ Gerson Meza Mori: https://orcid.org/0000-0002-9754-6725. E-mail: gersonmmori@gmail.com \\ Elgar Barboza: https://orcid.org/0000-0002-9628-8138. E-mail: ebarboza@indes-ces.edu.pe \\ Nilton B. Rojas Briceño: https://orcid.org/0000-0002-5352-6140. E-mail: nrojas@indes-ces.edu.pe \\ Cristóbal Torres Guzmán: https://orcid.org0000-0002-0932-7224 E-mail: cristobal.torres@untrm.edu.pe \\ Manuel Oliva: https://orcid.org/0000-0002-9670-0970. E-mail: soliva@indes-ces.edu.pe \\ Segundo G. Chavez-Quintana: https://orcid.org/0000-0002-0946-3445. E-mail: segundo.quintana@untrm.edu.pe \\ Rolando Salas López: https://orcid.org/0000-0003-2184-6761. E-mail: rsalas@indes-ces.edu.pe \\ C. Rocio López de la Lama: https://orcid.org/0000-0001-6696-5401. Email: rocio.lopezdelalama@gmail.com \\ Steven Sevillano-Ríos: http://orcid.org/0000-0002-0642-5224. E-mail: ssevillano@corbidi.org \\ Fausto Sarmiento: http://orcid.org/0000-0003-0501-6020. Email: fsarmien@uga.edu \\ *Autor corresponsal: eldeflorian@uga.edu
}

MATERIAL SUPLEMENTARIO 
Tabla S1. Cálculo del Índice de Efectividad de Manejo (IEM).

\begin{tabular}{|c|c|c|c|c|c|}
\hline Índice/Criterios & Indicadores & Copallín & $\begin{array}{c}\text { Hierba Buena - } \\
\text { Allpayacu }\end{array}$ & Tilacancha & Huaylla Belén \\
\hline \multirow{6}{*}{ Contexto } & IB & 3,8 & 3,1 & 3,8 & 2,9 \\
\hline & ISE & 3,6 & 3,0 & 2,7 & 2,6 \\
\hline & VD & 2,4 & 1,7 & 1,9 & 2,0 \\
\hline & PAP & 2,5 & 0,7 & 1,2 & 1,6 \\
\hline & $\mathrm{AM}$ & 2,0 & 0,7 & 2,0 & 1,6 \\
\hline & Media & 2,9 & 1,8 & 2,3 & 2,1 \\
\hline \multirow{5}{*}{ Planificación } & OB & 3,6 & 3,2 & 3,3 & 1,9 \\
\hline & $\mathrm{SL}$ & 2,5 & 2,7 & 1,8 & 2,5 \\
\hline & DSP & 3,7 & 3,0 & 3,3 & 2,7 \\
\hline & DSA & 3,2 & 0,9 & 2,1 & 2,1 \\
\hline & Media & 3,2 & 2,4 & 2,6 & 2,3 \\
\hline \multirow{5}{*}{ Insumo } & $\mathrm{AP}$ & 2,2 & 1,8 & 1,0 & 1,1 \\
\hline & $\mathrm{CI}$ & 2,6 & 2,1 & 1,5 & 1,2 \\
\hline & $\mathrm{IE}$ & 2,4 & 1,9 & 1,9 & 1,2 \\
\hline & FI & 2,0 & 1,5 & 1,8 & 1,3 \\
\hline & Media & 2,3 & 1,8 & 1,6 & 1,2 \\
\hline \multirow{4}{*}{ Procesos } & PM & 2,2 & 1,1 & 1,3 & 1,4 \\
\hline & TDM & 4,0 & 3,1 & 2,3 & 1,4 \\
\hline & IME & 2,4 & 1,2 & 1,4 & 1,0 \\
\hline & Media & 2,9 & 1,8 & 1,7 & 1,3 \\
\hline Resultados & $\mathrm{RS}$ & 2,6 & 1,3 & 1,2 & 1,3 \\
\hline \multicolumn{2}{|c|}{ IEM } & 2,77 & 1,85 & 1,86 & 1,63 \\
\hline
\end{tabular}

\section{Contexto}

IB Importancia biológica

ISE Importancia socio-económica

VD Vulnerabilidad

PAP Políticas de las áreas protegidas

AM Ambiente político

\section{Planificación}

OB Objetivos

SL Seguridad legal

DSP Diseño del sitio y planificación

DSA Diseño del sub-sistema de áreas protegidas

\section{Insumo}

AP Asignación de personal

CI Comunicación e información

IE Infraestructura y equipo

FI Finanzas

Procesos

PM Planificación del manejo

TDM Toma de decisiones para el manejo

IME Investigación, monitoreo y evaluación

Resultados

RS Resultados 
Tabla S2. Cálculo del subíndice de representatividad de elevación (Cle) para el Índice de Efectividad de Diseño (IED).

\begin{tabular}{|c|c|c|c|c|c|c|c|}
\hline $\begin{array}{l}\text { Nombre de } \\
\text { las Áreas de } \\
\text { Conservación } \\
\text { Privada (ACPs) }\end{array}$ & $\begin{array}{c}\text { Clases de } \\
\text { altitud (msnm) }\end{array}$ & $\begin{array}{c}\text { Superficie } \\
\text { (ha) por rango } \\
\text { altitudinal } \\
\text { de ACPs - } \\
\text { Departamento }\end{array}$ & $\begin{array}{l}\% \text { de superficie } \\
\text { por rango } \\
\text { altitudinal } \\
\text { de ACPs - } \\
\text { Departamento }\end{array}$ & $\begin{array}{c}\text { Superficie } \\
\text { (ha) por rango } \\
\text { altitudinal de } \\
\text { ACP }\end{array}$ & $\begin{array}{c}\% \text { de } \\
\text { superficie } \\
\text { (ha) por } \\
\text { rango } \\
\text { altitudinal de } \\
\text { ACP } \\
\end{array}$ & & Cle \\
\hline \multirow{3}{*}{$\begin{array}{l}\text { Abra Patricia - } \\
\text { Alto Nieva }\end{array}$} & $1500-2000$ & $13.964,42$ & 9,42 & 327,43 & 23,13 & 2,45 & \multirow{3}{*}{1,86} \\
\hline & $2000-2500$ & $36.410,48$ & 24,57 & $1.071,62$ & 75,69 & 3,08 & \\
\hline & $2500-3000$ & $45.453,89$ & 30,67 & 16,69 & 1,18 & 0,04 & \\
\hline \multirow{2}{*}{ Arroyo Negro } & $2500-3000$ & $45.453,89$ & 30,67 & 71,01 & 45,40 & 1,48 & \multirow{2}{*}{1,85} \\
\hline & $3000-3500$ & $36.367,35$ & 24,54 & 85,40 & 54,60 & 2,23 & \\
\hline \multirow{2}{*}{ Berlín } & $2000-2500$ & $36.410,48$ & 24,57 & 42,10 & 71,36 & 2,90 & \multirow{2}{*}{1,92} \\
\hline & $2500-3000$ & $45.453,89$ & 30,67 & 16,90 & 28,64 & 0,93 & \\
\hline \multirow{3}{*}{$\begin{array}{l}\text { Bosque de } \\
\text { Palmeras de } \\
\text { la Comunidad } \\
\text { Campesina Taulia } \\
\text { Molinopampa }\end{array}$} & $2000-2500$ & $36.410,48$ & 24,57 & $3.883,32$ & 35,56 & 1,45 & \multirow{3}{*}{1,19} \\
\hline & $2500-3000$ & $45.453,89$ & 30,67 & $6.903,35$ & 63,21 & 2,06 & \\
\hline & $3000-3500$ & $36.367,35$ & 24,54 & 134,17 & 1,23 & 0,05 & \\
\hline \multirow{2}{*}{ Cavernas de Leo } & $1000-1500$ & $2.416,65$ & 1,63 & 3,12 & 24,99 & 15,33 & \multirow{2}{*}{11,64} \\
\hline & $1500-2000$ & $13.964,42$ & 9,42 & 9,38 & 75,01 & 7,96 & \\
\hline \multirow{4}{*}{$\begin{array}{l}\text { Comunal San } \\
\text { Pablo-Catarata } \\
\text { Gocta }\end{array}$} & $1500-2000$ & $13.964,42$ & 9,42 & 249,88 & 9,60 & 1,02 & \multirow{4}{*}{1,06} \\
\hline & $2000-2500$ & $36.410,48$ & 24,57 & 440,38 & 16,91 & 0,69 & \\
\hline & $2500-3000$ & $45.453,89$ & 30,67 & $1.520,17$ & 58,39 & 1,90 & \\
\hline & $3000-3500$ & $36.367,35$ & 24,54 & 393,15 & 15,10 & 0,62 & \\
\hline \multirow{4}{*}{ Copal Cuilungo } & $1500-2000$ & $13.964,42$ & 9,42 & 232,53 & 9,04 & 0,96 & \multirow{4}{*}{1,15} \\
\hline & $2000-2500$ & $36.410,48$ & 24,57 & $2.049,30$ & 79,64 & 3,24 & \\
\hline & $2500-3000$ & $45.453,89$ & 30,67 & 235,55 & 9,15 & 0,30 & \\
\hline & $3000-3500$ & $36.367,35$ & 24,54 & 55,70 & 2,16 & 0,09 & \\
\hline \multirow{5}{*}{ Copallín } & $1500-2000$ & $13.964,42$ & 9,42 & 64,98 & 0,56 & 0,06 & \multirow{5}{*}{0,79} \\
\hline & $2000-2500$ & $36.410,48$ & 24,57 & $1.836,63$ & 15,90 & 0,65 & \\
\hline & $2500-3000$ & $45.453,89$ & 30,67 & $5.176,20$ & 44,82 & 1,46 & \\
\hline & $3000-3500$ & $36.367,35$ & 24,54 & $4.176,56$ & 36,16 & 1,47 & \\
\hline & $3500-4000$ & $12.458,73$ & 8,41 & 294,84 & 2,55 & 0,30 & \\
\hline \multirow{6}{*}{$\begin{array}{l}\text { Hierba Buena - } \\
\text { Allpayacu }\end{array}$} & $2000-2500$ & $36.410,48$ & 24,57 & 27,40 & 1,20 & 0,05 & \multirow{3}{*}{1,11} \\
\hline & $2500-3000$ & $45.453,89$ & 30,67 & $2.088,60$ & 91,52 & 2,98 & \\
\hline & $3000-3500$ & $36.367,35$ & 24,54 & 166,12 & 7,28 & 0,30 & \\
\hline & $1500-2000$ & $13.964,42$ & 9,42 & 27,10 & 0,43 & 0,05 & \multirow{5}{*}{0,86} \\
\hline & $2000-2500$ & $36.410,48$ & 24,57 & 656,81 & 10,36 & 0,42 & \\
\hline & $2500-3000$ & $45.453,89$ & 30,67 & $1.934,25$ & 30,52 & 1,00 & \\
\hline \multirow{2}{*}{$\begin{array}{l}\text { Hierba Buena- } \\
\text { Allpayacku }\end{array}$} & $3000-3500$ & $36.367,35$ & 24,54 & $3.366,07$ & 53,11 & 2,16 & \\
\hline & $3500-4000$ & $12.458,73$ & 8,41 & 354,20 & 5,59 & 0,66 & \\
\hline & $2500-3000$ & $45.453,89$ & 30,67 & 393,36 & 34,49 & 1,12 & \\
\hline Huiquilla & $3000-3500$ & $36.367,35$ & 24,54 & 690,78 & 60,57 & 2,47 & 1,39 \\
\hline & $3500-4000$ & $12.458,73$ & 8,41 & 56,41 & 4,95 & 0,59 & \\
\hline La Pampa del & $1500-2000$ & $13.964,42$ & 9,42 & $2.294,60$ & 82,63 & 8,77 & 474 \\
\hline Burro & $2000-2500$ & $36.410,48$ & 24,57 & 482,36 & 17,37 & 0,71 & 4,74 \\
\hline & $2000-2500$ & $36.410,48$ & 24,57 & 687,17 & 3,93 & 0,16 & \\
\hline Llamapampa - La & $2500-3000$ & $45.453,89$ & 30,67 & $6.013,90$ & 34,36 & 1,12 & 007 \\
\hline Jalca & $3000-3500$ & $36.367,35$ & 24,54 & $10.643,69$ & 60,81 & 2,48 & 0,97 \\
\hline & $3500-4000$ & $12.458,73$ & 8,41 & 158,18 & 0,90 & 0,11 & \\
\hline & $1000-1500$ & $2.416,65$ & 1,63 & 270,37 & 0,59 & 0,36 & \\
\hline & $1500-2000$ & $13.964,42$ & 9,42 & $7.301,59$ & 15,87 & 1,68 & \\
\hline & $2000-2500$ & $36.410,48$ & 24,57 & $17.594,26$ & 38,25 & 1,56 & \\
\hline Los Chilchos & $2500-3000$ & $45.453,89$ & 30,67 & $12.402,50$ & 26,96 & 0,88 & 0,80 \\
\hline & $3000-3500$ & $36.367,35$ & 24,54 & $7.097,00$ & 15,43 & 0,63 & \\
\hline & $3500-4000$ & $12.458,73$ & 8,41 & $1.277,25$ & 2,78 & 0,33 & \\
\hline & $>4000$ & 988,41 & 0,67 & 57,02 & 0,12 & 0,19 & \\
\hline
\end{tabular}




\begin{tabular}{|c|c|c|c|c|c|c|c|}
\hline $\begin{array}{l}\text { Nombre de } \\
\text { las Áreas de } \\
\text { Conservación } \\
\text { Privada (ACPs) }\end{array}$ & $\begin{array}{c}\text { Clases de } \\
\text { altitud (msnm) }\end{array}$ & $\begin{array}{c}\text { Superficie } \\
\text { (ha) por rango } \\
\text { altitudinal } \\
\text { de ACPs - } \\
\text { Departamento }\end{array}$ & $\begin{array}{l}\% \text { de superficie } \\
\text { por rango } \\
\text { altitudinal } \\
\text { de ACPs - } \\
\text { Departamento }\end{array}$ & $\begin{array}{c}\text { Superficie } \\
\text { (ha) por rango } \\
\text { altitudinal de } \\
\text { ACP }\end{array}$ & $\begin{array}{c}\% \text { de } \\
\text { superficie } \\
\text { (ha) por } \\
\text { rango } \\
\text { altitudinal de } \\
\text { ACP } \\
\end{array}$ & & Cle \\
\hline $\begin{array}{l}\text { Milpuj - La } \\
\text { Heredad }\end{array}$ & $1500-2000$ & $13.964,42$ & 9,42 & 16,57 & 100,00 & 10,61 & 10,61 \\
\hline \multirow{6}{*}{$\begin{array}{l}\text { Monte Puyo } \\
\text { (Bosque de } \\
\text { Nubes) }\end{array}$} & $1000-1500$ & $2.416,65$ & 1,63 & 563,60 & 3,49 & 2,14 & \multirow{6}{*}{1,10} \\
\hline & $1500-2000$ & $13.964,42$ & 9,42 & $1.953,01$ & 12,09 & 1,28 & \\
\hline & $2000-2500$ & $36.410,48$ & 24,57 & $6.061,30$ & 37,52 & 1,53 & \\
\hline & $2500-3000$ & $45.453,89$ & 30,67 & $5.536,44$ & 34,28 & 1,12 & \\
\hline & $3000-3500$ & $36.367,35$ & 24,54 & $2.011,73$ & 12,45 & 0,51 & \\
\hline & $3500-4000$ & $12.458,73$ & 8,41 & 26,92 & 0,17 & 0,02 & \\
\hline \multirow{3}{*}{ San Antonio } & $1500-2000$ & $13.964,42$ & 9,42 & 104,12 & 29,13 & 3,09 & \multirow{3}{*}{1,96} \\
\hline & $2000-2500$ & $36.410,48$ & 24,57 & 212,51 & 59,46 & 2,42 & \\
\hline & $2500-3000$ & $45.453,89$ & 30,67 & 40,76 & 11,40 & 0,37 & \\
\hline \multirow{8}{*}{$\begin{array}{l}\text { San Pedro de } \\
\text { Chuquibamba }\end{array}$} & $500-1000$ & 158,84 & 0,11 & 158,86 & 0,81 & 7,58 & \multirow{8}{*}{3,47} \\
\hline & $1000-1500$ & $2.416,65$ & 1,63 & $1.579,62$ & 8,08 & 4,95 & \\
\hline & $1500-2000$ & $13.964,42$ & 9,42 & $1.383,58$ & 7,07 & 0,75 & \\
\hline & $2000-2500$ & $36.410,48$ & 24,57 & $1.365,00$ & 6,98 & 0,28 & \\
\hline & $2500-3000$ & $45.453,89$ & 30,67 & $1.635,21$ & 8,36 & 0,27 & \\
\hline & $3000-3500$ & $36.367,35$ & 24,54 & $2.205,27$ & 11,27 & 0,46 & \\
\hline & $3500-4000$ & $12.458,73$ & 8,41 & $10.292,08$ & 52,62 & 6,26 & \\
\hline & $>4000$ & 988,41 & 0,67 & 940,38 & 4,81 & 7,21 & \\
\hline \multirow[t]{2}{*}{ Tilacancha } & $2500-3000$ & $45.453,89$ & 30,67 & $1.463,21$ & 21,52 & 0,70 & \multirow{2}{*}{1,95} \\
\hline & $3000-3500$ & $36.367,35$ & 24,54 & $5.337,27$ & 78.48 & 3.20 & \\
\hline
\end{tabular}

Tabla S3. Cálculo del Índice de Efectividad Ecológico (IEE).

\begin{tabular}{|l|c|c|c|c|c|c|c|c|}
\hline \multicolumn{2}{|l|}{ Valores usados para el cálculo del Índice de efectividad ecológico } \\
\hline ACP & $\begin{array}{l}\text { TC- } \\
\text { ACP }\end{array}$ & $\begin{array}{l}\text { TC-Área } \\
\text { circundante }\end{array}$ & $\begin{array}{l}\text { ACP- } \\
\text { AC }\end{array}$ & $\begin{array}{l}\text { Valor } \\
\text { estandarizado }\end{array}$ & $\begin{array}{l}\text { \% ST } \\
\mathbf{2 0 1 8}\end{array}$ & $\begin{array}{l}\text { TC ACP } \\
\mathbf{2 0 1 1 - 2 0 1 8}\end{array}$ & $\begin{array}{l}\text { TC- } \\
\text { Ecorregión }\end{array}$ & $\begin{array}{l}\text { Índice de } \\
\text { efectividad } \\
\text { ecológico }\end{array}$ \\
\hline $\begin{array}{l}\text { Hierba Buena- } \\
\text { Allpayacku }\end{array}$ & 0,13 & 1,31 & $-1,18$ & 1 & 0,01 & 0,13 & 1 & 2,14 \\
\hline $\begin{array}{l}\text { Hualla Belen- } \\
\text { Colcamar }\end{array}$ & 0,18 & 0,53 & $-0,35$ & 1 & 0,01 & 0,18 & 1 & 2,19 \\
\hline Tilacancha & 0,16 & $-1,91$ & 2,07 & 0 & 0 & 0,31 & 1 & 1,31 \\
\hline Copallín & 0,03 & 0,19 & $-0,16$ & 1 & 0,02 & 0,02 & 1,0 & 2,04 \\
\hline
\end{tabular}

ACP Área de Conservación Privada

TC Tasa de cambio de superficie transformada

ACP-AC Diferencia de la tasa de cambio de la superficie transformada en el ACP y su área circundante

ST Superficie Transformada 

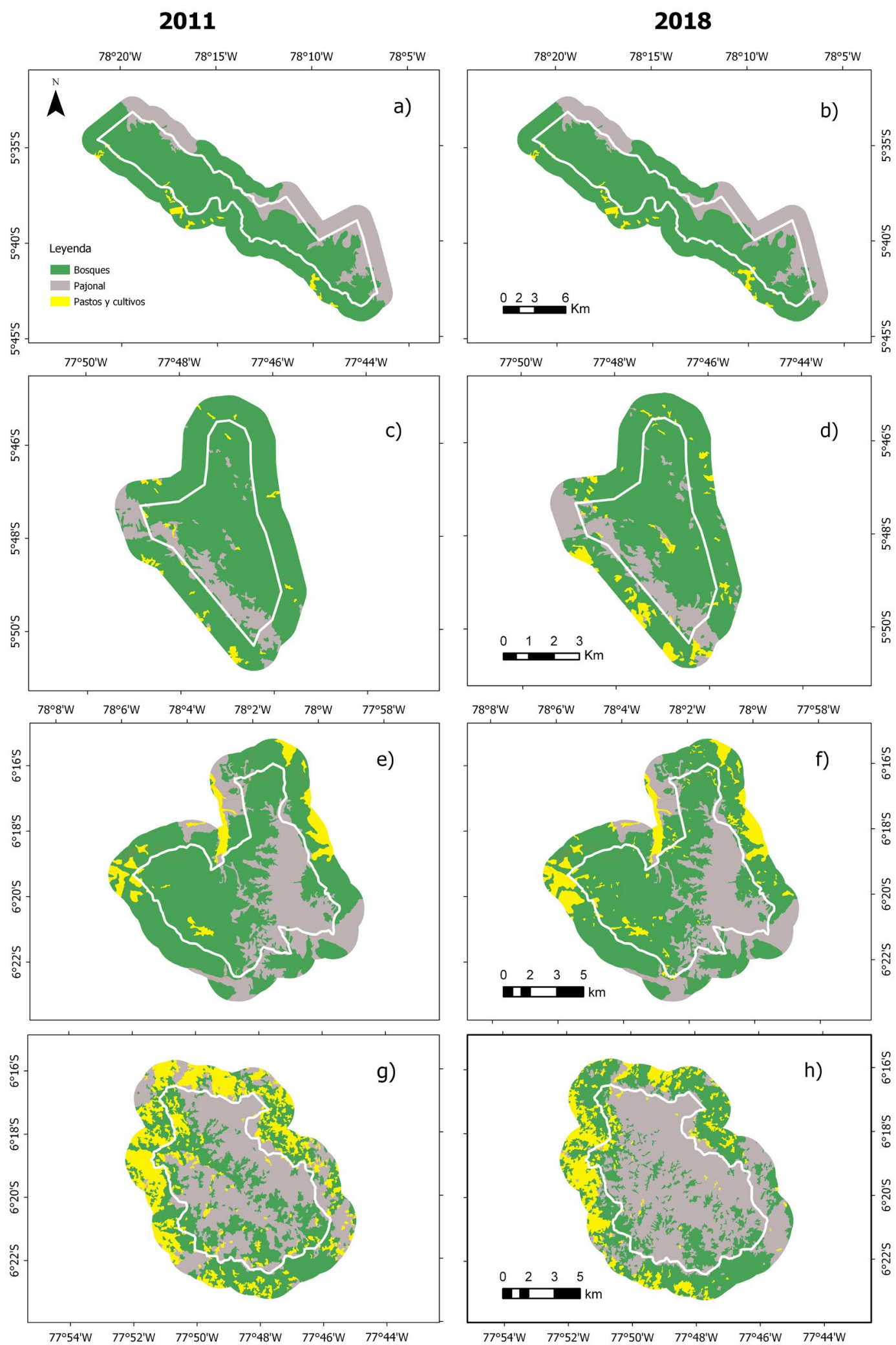

Figura S1. Mapas de Cobertura y Uso del Suelo (CUS) generados para el cálculo de Índice de Efectividad Ecológica (IEE) mediante tasas de cambio en tres clases de CUS. ACP Copallin (a,b), ACP Hierba Buena- Allpayacku (c,d), ACP Hualla Belén Colcamar (e,g), ACP Tilacancha 
S6 • E. DELGADO, G. MEZA, E. BARBOZA, N. ROJAS, C. TORRES, M. OLIVA, S. CHAVEZ, R. SALAS, R. LÓPEZ, C. STEVEN, F. SARMIENTO

Tabla S4. Cálculo del Índice de Efectividad Compuesto (IEC).

\begin{tabular}{|l|c|c|c|c|c|c|c|c|}
\hline \multicolumn{1}{|c|}{ ACP } & $\begin{array}{c}\text { Índice de } \\
\text { efectividad } \\
\text { ecológico } \\
\text { IEE }\end{array}$ & $\begin{array}{c}\text { Peso 1 } \\
\boldsymbol{x}\end{array}$ & $\begin{array}{c}\text { Índice de } \\
\text { efectividad } \\
\text { del diseño } \\
\text { IED }\end{array}$ & $\begin{array}{c}\text { Peso 2 } \\
\boldsymbol{y}\end{array}$ & $\begin{array}{c}\text { Índice de } \\
\text { efectividad } \\
\text { del manejo } \\
\text { IEM }\end{array}$ & $\begin{array}{c}\text { Peso 3 } \\
\boldsymbol{z}\end{array}$ & IEC1 & IEC2 \\
\hline Copallín & 0,51042 & 0,4778 & 0,08770 & 0,1556 & 0,69250 & 0,3667 & 0,511 & 1,291 \\
\hline $\begin{array}{l}\text { Hierba Buena- } \\
\text { Allpayacku }\end{array}$ & 0,53483 & 0,4778 & 0,09190 & 0,1556 & 0,46250 & 0,3667 & 0,439 & 1,089 \\
\hline $\begin{array}{l}\text { Hualla Belen- } \\
\text { Colcamar }\end{array}$ & 0,54823 & 0,4778 & 0,09420 & 0,1556 & 0,40750 & 0,3667 & 0,426 & 1,050 \\
\hline Tilacancha & 0,32799 & 0,4778 & 0,05636 & 0,1556 & 0,46500 & 0,3667 & 0,336 & 0,849 \\
\hline Mínimo & 0,25 & 0,4778 & 0,06787 & 0,1556 & 0,25 & 0,3667 & 0,222 & 0,568 \\
\hline Máximo & 1 & 0,4778 & 1 & 0,1556 & 1 & 0,3667 & 1,000 & 3,000 \\
\hline
\end{tabular}

